\title{
Diffusion in tilted periodic potentials: Enhancement, universality, and scaling
}

\author{
P. Reimann, ${ }^{1}$ C. Van den Broeck, ${ }^{2}$ H. Linke, ${ }^{3}$ P. Hänggi, ${ }^{1}$ J. M. Rubi, ${ }^{4}$ and A. Pérez-Madrid ${ }^{4}$ \\ ${ }^{1}$ Institut für Physik, Universität Augsburg, Universitätsstrasse 1, D-86135 Augsburg, Germany \\ ${ }^{2}$ Limburgs Universitair Centrum, B-3590 Diepenbeek, Belgium \\ ${ }^{3}$ Department of Physics, University of Oregon, Eugene, Oregon 97403-1274 \\ ${ }^{4}$ Department de Fisica Fonamental, Facultat de Fisica, Universitat de Barcelona, Diagonal 647, E-08028 Barcelona, Spain
}

(Received 4 September 2001; published 12 February 2002)

\begin{abstract}
An exact analytical expression for the effective diffusion coefficient of an overdamped Brownian particle in a tilted periodic potential is derived for arbitrary potentials and arbitrary strengths of the thermal noise. Near the critical tilt (threshold of deterministic running solutions) a scaling behavior for weak thermal noise is revealed and various universality classes are identified. In comparison with the bare (potential-free) thermal diffusion, the effective diffusion coefficient in a critically tilted periodic potential may be, in principle, arbitrarily enhanced. For a realistic experimental setup, an enhancement by 14 orders of magnitude is predicted so that thermal diffusion should be observable on a macroscopic scale at room temperature.
\end{abstract}

DOI: 10.1103/PhysRevE.65.031104

PACS number(s): 05.40.-a, 05.60.-k, 02.50.Ey

\section{INTRODUCTION}

Within the realm of thermal equilibrium, the overdamped force-free thermal diffusion of a single Brownian particle as considered, e.g., by Einstein in Ref. [1], is always reduced when an additional periodic potential is switched on [2], and it is therefore tempting to conjecture a qualitatively similar behavior at least for time-independent nonequilibrium systems. A first main conclusion of our present explorations is that the opposite is the case: The effective diffusion coefficient of a Brownian particle in a periodic potential that is driven away from equilibrium by a static "tilting force" can become arbitrarily much larger than in the presence of thermal noise alone. A striking consequence of our finding is the possibility to observe thermal diffusion of macroscopic particles on macroscopic time and length scales at roomtemperature in appropriate tilted periodic structures.

A second main result of our present paper are scaling relations for the diffusion coefficient that become asymptotically exact in the limit of weak thermal fluctuations and small deviations from the critical tilt (i.e., the threshold at which deterministically running solutions set in). Furthermore, the asymptotic behavior of the diffusion leads to a classification into different universality classes with scaling exponent and scaling function depending on the characteristics of the potential at the critical tilt. These conceptsscaling and universality - are a recurrent theme in many branches of statistical physics, such as, e.g., critical phenomena, hydrodynamics, or low-dimensional nonlinear dynamics [3]. Most closely related to our present findings are the scaling and universality phenomena as observed in the context of so-called deterministic diffusion [4-8], especially in the presence of noise [9-11], and of noisy systems at a saddlenode bifurcation (e.g., relaxation oscillations) $[12,13]$.

At the basis of all our above mentioned findings is an exact analytical expression for the diffusion coefficient, applicable to arbitrary periodic potentials, arbitrary tilts, and arbitrary strengths of the thermal noise [see Eq. (22) below].

Besides describing a real Brownian particle, thermal diffusion in a tilted periodic potential, as we will consider it here, is of relevance in numerous other contexts (see also Chap. 11 in Ref. [14]), such as Josephson junctions [15], the motion of fluxons in superconductors [16], rotating dipoles in external fields [17], the rotation of molecules in solids [18], superionic conductors [19], charge density waves [20], synchronization phenomena [21] in electrical circuits as described by the Adler equation or in phase locked loops [22], mode locking in laser gyroscopes [23], plasma accelerators [24], diffusion of atoms and molecules on crystal surfaces [25], particle separation by electrophoresis [26], biophysical processes such as neural activity [27] and intracellular transport [28], and possibly also for the explanation of the matterantimatter asymmetry of the universe [29]. Also worth noting is the fact that the Brownian motion in a "traveling periodic potential" (pump) of the form $V_{0}(x-v t)$ can be readily mapped onto a static tilted periodic potential [30]. Moreover, our results near criticality (marginal stability) are universal for general dynamical systems close to a saddle-node bifurcation $[12,13]$, such as for instance relaxation oscillations.

With the present work we continue and explain in more detail our brief account in Ref. [31]. The organization of the paper is as follows: In Sec. II we introduce the model and the basic quantities of interest, namely, the average particle current and the effective diffusion coefficient. In Sec. III we derive as our first main result the relation (15) between the diffusion coefficient and the first two moments of the first passage time distribution. As a consequence, the closed analytical expression (22) can be inferred. Section IV is devoted to the exploration of universality and scaling properties of the diffusion coefficient near the threshold of deterministically running solutions (critically tilted periodic potentials), predicting a giant enhancement of the free thermal diffusion under suitable conditions. The latter result is exemplified in Sec. V for the special case of a mechanical Brownian particle that moves in a critically tilted geometrical profile. The summary and discussion of our findings is presented in Sec. VI.

\section{MODEL}

We consider the following model for the overdamped Brownian motion of a particle with coordinate $x(t)$ : 


$$
\begin{gathered}
\eta \dot{x}(t)=-V^{\prime}(x(t))+\xi(t), \\
V(x):=V_{0}(x)-x F,
\end{gathered}
$$

where $\eta$ is the viscous friction coefficient (static mobility) of the particle and where the total potential $V(x)$ consists of a periodic part $V_{0}(x)$ with period $L$,

$$
V_{0}(x+L)=V_{0}(x),
$$

and a homogeneous, static "tilting force" $F$. Further, thermal fluctuations are modeled as usual [32] by Gaussian white noise with zero average and correlation

$$
\langle\xi(t) \xi(s)\rangle=2 \eta k T \delta(t-s),
$$

where $T$ is the temperature, $k$ is the Boltzmann constant, and $\langle\cdots\rangle$ indicates the (nonequilibrium) average over a statistical ensemble of realizations in Eq. (1). Finally, as compared to a full fledged Newtonian equation of motion, an inertia term $m \ddot{x}(t)$ is missing on the left-hand side of Eq. (1). In other words, this inertia term is assumed to have a negligibly small effect in comparison with the other forces appearing in Eq. (1), hence the name overdamped motion [14,32].

A first basic quantity of interest is the average particle current in the long-time limit (i.e., after transients due to initial conditions have died out)

$$
\langle\dot{x}\rangle:=\lim _{t \rightarrow \infty} \frac{\langle x(t)\rangle}{t} .
$$

The analytical solution for this current goes back to Stratonovich [33] and has subsequently been rederived many times [see, e.g., Chap. 11 in Ref. [14]; the explicit formula will be given in Eq. (18) below]. The fact that such an exact closed solution can be given without any further restrictions in the model (1) is rather exceptional and has given this model the status of a "hydrogen atom" in the context of Brownian motion theory.

In our present study, the quantity of central interest will be the effective diffusion coefficient

$$
D:=\lim _{t \rightarrow \infty} \frac{\left\langle x^{2}(t)\right\rangle-\langle x(t)\rangle^{2}}{2 t} .
$$

Exact analytical results are known in two special cases. First, in the absence of the periodic potential $V_{0}(x)$ in Eq. (1) a straightforward calculation yields the so-called Einstein relation

$$
D=k T / \eta=: D_{0} \quad \text { if } \quad V_{0}^{\prime}(x) \equiv 0
$$

for arbitrary values of the static tilt $F$ [34]. Second, in the absence of a tilt $F$, the following analytic prediction for the diffusion coefficient is due to $[2,35]$

$$
D=\frac{D_{0}}{\int_{0}^{L} \frac{d x}{L} e^{V_{0}(x) / k T} \int_{0}^{L} \frac{d y}{L} e^{-V_{0}(y) / k T}} \text { if } F=0 .
$$

It has been furthermore demonstrated in Ref. [2] that $D$ $\leqslant D_{0}$, basically by applying the Cauchy-Schwartz inequality to the denominator in Eq. (8).

The evaluation of the diffusion coefficient (6) in the presence of both an arbitrary tilt $F$ and an arbitrary periodic potential $V_{0}(x)$ is not obvious. One of the main objectives of the present work is the derivation of such a general, exact diffusion formula, analogous to Stratonovich's result for the current.

\section{EVALUATION OF THE DIFFUSION COEFFICIENT}

In order to evaluate the diffusion coefficient (6) we will take advantage of another quantity that is analytically known for the model (1), namely, the moments of the first passage time. To define these quantities, we consider the stochastic process (1) with an arbitrary but fixed seed $x(0)=x_{0}$ and we denote by $t\left(x_{0} \rightarrow b\right)$ the time until an arbitrary but fixed point $b$ is reached for the first time. Then the $n$th moment of the first passage time is the statistical average

$$
T_{n}\left(x_{0} \rightarrow b\right):=\left\langle t^{n}\left(x_{0} \rightarrow b\right)\right\rangle .
$$

In what follows, we will temporarily restrict ourselves to the case $F>0$ and $b>x_{0}$, since otherwise the averages in Eq. (9) may diverge. Then, for the one-dimensional dynamics (1), these moments of the first passage time are given by the well-known closed analytical recursion (see, e.g., Sec. 7 in Ref. [32] and further references therein)

$$
\begin{aligned}
T_{n}\left(x_{0} \rightarrow b\right)= & \frac{n}{D_{0}} \int_{x_{0}}^{b} d x e^{V(x) / k T} \int_{-\infty}^{x} d y e^{-V(y) / k T} \\
& \times T_{n-1}(y \rightarrow b)
\end{aligned}
$$

for $n=1,2 \ldots$ and with $T_{0}(y \rightarrow b) \equiv 1$. Note that the convergence of the integrals in Eq. (10) is guaranteed by our assumption that $F>0$ and $b>x_{0}$. In principle, it is quite plausible that all properties of the stochastic process (1) should be expressible in terms of the moments (10) and, in this sense, available in closed analytical form. In practice, the explicit connection between a given quantity of interest and the moments is, however, not at all obvious.

We now come to the first main point of our paper, namely, the derivation of an exact expression for the diffusion coefficient $D$ in terms of the mean first passage time $T_{1}\left(x_{0}\right.$ $\rightarrow b$ ) and the so-called first passage time dispersion

$$
\begin{aligned}
\Delta T_{2}\left(x_{0} \rightarrow b\right) & :=\left\langle t^{2}\left(x_{0} \rightarrow b\right)\right\rangle-\left\langle t\left(x_{0} \rightarrow b\right)\right\rangle^{2} \\
& =T_{2}\left(x_{0} \rightarrow b\right)-\left[T_{1}\left(x_{0} \rightarrow b\right)\right]^{2} .
\end{aligned}
$$

To this end, we denote by $a$ an arbitrary point between $x_{0}$ and $b$. Then the time $t\left(x_{0} \rightarrow b\right)$ that the stochastic process in Eq. (1) needs to travel from $x_{0}$ to $b$ can be decomposed into the time to travel from $x_{0}$ to $a$, plus the time to travel from $a$ to $b$. For a white noise driven process (1), the latter two times are statistically independent of each other [36]. Further, since the process (1) is homogeneous in time, all statistical properties of $t\left(x_{0} \rightarrow b\right)$ are exactly the same as those of $t\left(x_{0} \rightarrow a\right)+t(a \rightarrow b)$ with $t\left(x_{0} \rightarrow a\right)$ and $t(a \rightarrow b)$ being sta- 
tistically independent of each other. It then follows readily from the definitions in Eqs. (9) and (11) that the mean first passage time $T_{1}\left(x_{0} \rightarrow b\right)$ and the dispersion $\Delta T_{2}\left(x_{0} \rightarrow b\right)$ are additive quantities, i.e.,

$$
\begin{gathered}
T_{1}\left(x_{0} \rightarrow b\right)=T_{1}\left(x_{0} \rightarrow a\right)+T_{1}(a \rightarrow b), \\
\Delta T_{2}\left(x_{0} \rightarrow b\right)=\Delta T_{2}\left(x_{0} \rightarrow a\right)+\Delta T_{2}(a \rightarrow b) .
\end{gathered}
$$

As a second consequence it follows that $t\left(x_{0} \rightarrow x_{0}+l L\right)$ is statistically equivalent to a sum of $l$ independent, random variables $t\left(x_{0} \rightarrow x_{0}+L\right), \ldots, t\left(x_{0}+(l-1) L \rightarrow x_{0}+l L\right)$, and due to the periodicity (3), they are identically distributed. Invoking the central limit theorem, the distribution of the first passage times $t\left(x_{0} \rightarrow x_{0}+l L\right)$ thus approaches (for large l) a Gaussian distribution with mean value $l T_{1}\left(x_{0} \rightarrow x_{0}+L\right)$ and variance $l \Delta T_{2}\left(x_{0} \rightarrow x_{0}+L\right)$.

Next, we introduce a discrete set of "coarse-grained states" along the $x$ axis $\left\{x_{m}:=x_{0}+m l L\right\}_{m=-\infty}^{\infty}$ with mutual distance $l L$, where $l$ is a large but fixed integer [37]. The process $x(t)$ is said to be in a certain "state" from the instant of time it hits the associated point $x_{m}$ until the moment it hits one of the adjacent neighboring points $x_{m \pm 1}$. It follows that both the current $\langle\dot{x}\rangle$ and the diffusion coefficient $D$ are identical for the original process $x(t)$ and its coarse-grained counterpart due to the long-time limits in the respective definitions (5) and (6). Next, we note that "backward transitions" $x_{m} \mapsto x_{m-1}$ require climbing up an "energy ramp" of height $l L F$ by thermal activation and are thus suppressed by a Boltzmann factor (barometric formula) $\exp \{-l L F / k T\}$ compared to $x_{m} \mapsto x_{m+1}$, i.e., "sliding down the ramp." For sufficiently large $l$ we, therefore, can safely neglect transitions $x_{m} \mapsto x_{m-1}$. The remaining "forward transitions" between neighboring "states" $x_{m}$ and $x_{m+1}$ are identically distributed random events [38] with a probability distribution that is identical to the first passage time distribution for the original process $x(t)$. In particular, the moments of the first passage time $T_{n}\left(x_{m} \rightarrow x_{m+1}\right)$ are thus identical for the original process $x(t)$ and its coarse-grained counterpart. On the other hand, we have seen above that for sufficiently large $l$, all these moments and hence the entire coarse-grained process is completely fixed by the mean first passage time $T_{1}\left(x_{0} \rightarrow x_{0}\right.$ $+L)$ and the dispersion $\Delta T_{2}\left(x_{0} \rightarrow x_{0}+L\right)$. As our main conclusion we thus find that if two processes (1) yield the same values of $T_{1}\left(x_{0} \rightarrow x_{0}+L\right)$ and $\Delta T_{2}\left(x_{0} \rightarrow x_{0}+L\right)$ then $\langle\dot{x}\rangle$ and $D$ will also be the same in the two cases.

With the above construction at our disposal, we may conclude [31] that

$$
\begin{gathered}
\langle\dot{x}\rangle=\frac{L}{T_{1}\left(x_{0} \rightarrow x_{0}+L\right)}, \\
D=\frac{L^{2}}{2} \frac{\Delta T_{2}\left(x_{0} \rightarrow x_{0}+L\right)}{\left[T_{1}\left(x_{0} \rightarrow x_{0}+L\right)\right]^{3}} .
\end{gathered}
$$

The proof of these relations follows from the consideration of the special case with a potential $V_{0}(x) \equiv 0$ in Eq. (1), implying $\langle\dot{x}\rangle=F / \eta$ and Eq. (7). The evaluation of $T_{1}\left(x_{0}\right.$ $\left.\rightarrow x_{0}+L\right)$ and $\Delta T_{2}\left(x_{0} \rightarrow x_{0}+L\right)$ according to Eqs. (10) and
(11) is straightforward and one sees that the relations (14), (15) are indeed fulfilled. But from our conclusion at the end of the preceding paragraph, it now follows that Eqs. (14) and (15) are also satisfied for a process (1) with an arbitrary periodic potential $V_{0}(x)$. We remark that here and in the following, the reference point $x_{0}$ is arbitrary.

By introducing Eqs. (10) and (11) into Eq. (15) an analytical formula for $D$ is recovered within our so far used restriction that $F>0$ (otherwise several terms in this formula would diverge). To remove this restriction we first rewrite the $y$ integral in Eq. (10) with $n=1$ as

$$
\int_{-\infty}^{x} d y e^{-V(y) / k T}=\sum_{l=0}^{\infty} \int_{x-L}^{x} d y e^{-V(y-l L) / k T} .
$$

According to Eqs. (2) and (3) we have that $V(y-l L)$ $=V(y)+l L F$, so that a geometrical series arises in Eq. (16); it can be summed to yield

$$
\int_{-\infty}^{x} d y e^{-V(y) / k T}=\frac{\int_{x-L}^{x} d y e^{-V(y) / k T}}{1-e^{-L F / k T}} .
$$

Using Eq. (14) with Eq. (10) we thus recover Stratonovichi's formula for the particle current [33],

$$
\langle\dot{x}\rangle=\frac{1-e^{-L F / k T}}{\int_{x_{0}}^{x_{0}+L} \frac{d x}{L} I_{ \pm}(x)},
$$

where we have introduced

$$
\begin{aligned}
& I_{+}(x):=\frac{1}{D_{0}} e^{V(x) / k T} \int_{x-L}^{x} d y e^{-V(y) / k T}, \\
& I_{-}(x):=\frac{1}{D_{0}} e^{-V(x) / k T} \int_{x}^{x+L} d y e^{V(y) / k T},
\end{aligned}
$$

and where " $I_{ \pm}$" indicates that the index may be chosen to be either "+" or "-". The equivalence of these indices in Eq. (18) follows by interchanging in the denominator the order of the two integrations in combination with some additional steps (see also Appendix A). We remark that the wellestablished formula (18) can also be used, by reversing steps, as a derivation of Eq. (14). For later use, we also note that

$$
I_{ \pm}(x):=\int_{0}^{L} \frac{d z}{D_{0}} \exp \{ \pm[V(x)-V(x \mp z)] / k T\} .
$$

By similar manipulations as used in the derivation of Eq. (18) (the details of which are given in Appendix A), we obtain for the diffusion coefficient (15) a central result of this paper, namely [31],

$$
D=D_{0} \frac{\int_{x_{0}}^{x_{0}+L} \frac{d x}{L} I_{ \pm}(x) I_{+}(x) I_{-}(x)}{\left[\int_{x_{0}}^{x_{0}+L} \frac{d x}{L} I_{ \pm}(x)\right]^{3}}
$$


with arbitrary reference points $x_{0}$ and arbitrary indices in $I_{ \pm}$ both in the numerator and the denominator. So far, we have restricted ourselves tacitly to $F>0$. It is not difficult to see that our results above for both the current and the diffusion coefficient remain valid also for $F<0$. Moreover, for both of them, the limit $F \rightarrow 0$ does not give rise to any problems: In Eq. (18) one recovers the obvious result $\langle\dot{x}\rangle=0$ [28] and by observing that in Eqs. (19) and (20) the $y$ integral is $x$ independent one readily recovers Eq. (8) from Eq. (22).

In other words, the above closed expressions for both the current (18) and the diffusion (22) are exact analytical results for arbitrary periodic potentials $V_{0}(x)$ and arbitrary forces $F$. While the current formula has been known for more than 30 years, the corresponding compact expression for the diffusion coefficient has, to the best of our knowledge, been derived here for the first time (see also the discussion in Sec. VI).

For $V_{0}(x) \equiv 0$ one recovers from Eq. (22) by means of a straightforward calculation the Einstein relation (7). For nontrivial potentials $V_{0}(x)$, the above general analytical expression is still rather complicated. One may evaluate it numerically for arbitrary potentials $V_{0}(x)$, and one may try to simplify it analytically for some special limits. A particularly interesting such limit will be considered in the next section. We remark that an analytical discussion of other limits in Eq. (22) is also possible, but will not be pursued further here (see also the remark below on the weak noise limit).

As far as the numerical evaluation of our formula (22) is concerned, a representative example for the sine potential

$$
V_{0}(x)=U_{0} \sin (2 \pi x / L),
$$

is depicted in Fig. 1. The purpose of this figure is threefold. First, it confirms within the numerical accuracy of the simulations that our analytical prediction (22) is indeed exact. Second, it contains a comparison with a formula for the diffusion coefficient recently proposed by Constantini and Marchesoni [39] of the form

$$
D=k T \frac{d}{d F}\langle\dot{x}\rangle,
$$

with $\langle\dot{x}\rangle$ given by Eq. (18). This prediction is expected to capture the correct qualitative behavior of $D$ under rather general conditions and can be shown to become asymptotically exact in any of the three limits $F \rightarrow 0, F \rightarrow \infty$, $V_{0}(x) / k T \rightarrow 0$. As far as the quantitative behavior of $D$ under general conditions is concerned, our findings in Fig. 1 show that the formula from Eq. (24) is at most a rough approximation - see also the discussion at the end of the next section. Third, the most interesting feature in Fig. 1 is the resonancelike behavior of the diffusion coefficient around that value of the tilt $F$ for which the potential in Eq. (2) ceases to exhibit local extrema, which apparently gets more and more pronounced as the thermal noise strength $k T$ and thus the bare (force-free) diffusion coefficient $D_{0}=k T / \eta$ in Eq. (7) decreases. In the next section we will consider in more analytical detail this special limit of weak noise in combination with a tilt $F$ close to its critical value.

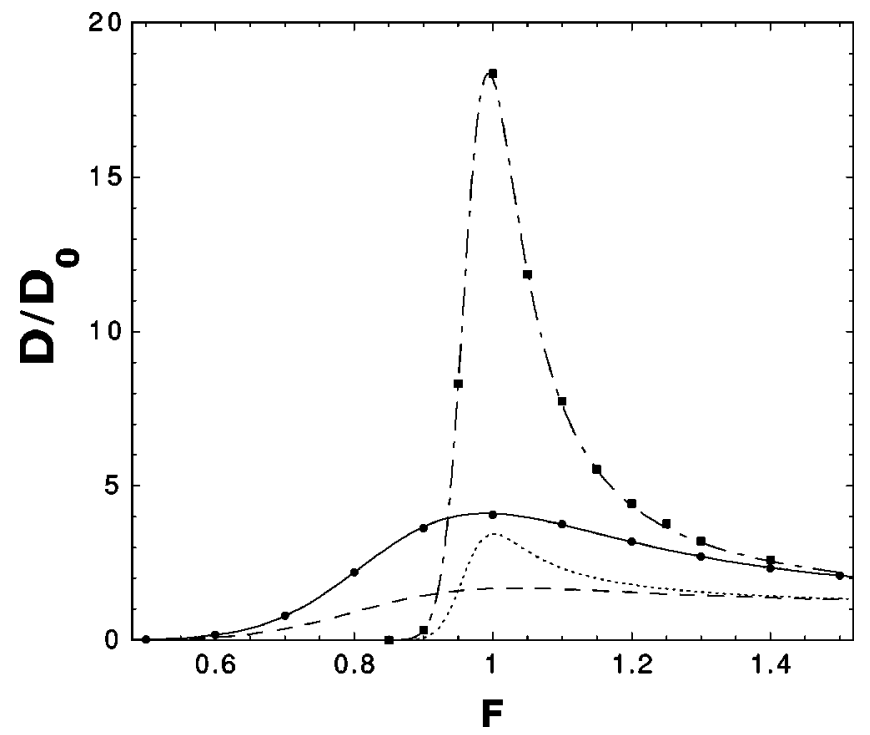

FIG. 1. Diffusion coefficient (6) versus the tilt $F$ for the overdamped model (1)-(4) with a sinusoidal periodic potential (23). Using dimensionless units, the parameter values are $\eta=U_{0}=1, L$ $=2 \pi, k T=D_{0}=0.1$. Note that the critical tilt [onset of deterministically running solutions in Eq. (1)] occurs at $F=F_{c}=1$. Solid line: analytical prediction (22). Filled dots: numerical simulations with an estimated relative uncertainty of 0.01 . Dashed line: analytical approximation (24), (18). Dashed-dotted line, filled squares, and dotted line: same as solid line, filled dots, and dashed line, respectively, but now for $k T=D_{0}=0.01$.

We finally remark that, for both numerical and analytical purposes, the reformulation (22) of the original analytical result in Eqs. (15) and (10) simplifies matters a lot in the weak noise limit, since only a very small (and usually quite evident) region of $z$ values then contributes significantly to the integrals in Eq. (21). The most involved case is the nearcritical regime treated in detail below. In any other case, the weak noise limit can be handled rather straightforwardly by standard steepest descent-type methods.

\section{UNIVERSALITY AND SCALING NEAR THE CRITICAL TILT}

We now turn to the case of a critically tilted periodic potential, i.e., we choose $F=F_{c}$ such that $V(x)$ in Eq. (2) exhibits a strictly monotonic behavior with the exception of exactly one inflection point within each period $L$. In other words, the tilted potential just ceases to display any local maxima and minima (saddle-node bifurcation [13]), corresponding to the threshold beyond which deterministically running solutions set in. When looking upon $x$ as a phaselike variable, we may also speak of relaxation oscillations in this context.

Without loss of generality we assume that $F_{c}>0$ (potential "tilted to the right") and that the inflection point is at $x=0$ (modulo $L$ ), i.e., $V(x)$ is strictly monotonically decreasing, $V^{\prime}(x)<0$, unless $x$ is a multiple of $L$. Next we assume that for

$$
F=F_{c}+\epsilon \text { with } F_{c}>0 \text { and } \epsilon \text { small, }
$$


the potential $V(x)$ can be written in the general form

$$
V(x)=-\mu \operatorname{sgn}(x)|x|^{q}-\epsilon x=-\mu x|x|^{q-1}-\epsilon x,
$$

in the vicinity of the critical point $x=0$. We furthermore restrict ourselves to the cases

$$
\mu>0, \quad q>1
$$

implying differentiability with

$$
V^{\prime}(x)=-\mu q|x|^{q-1}-\epsilon .
$$

In the remainder of the interval $[-L / 2, L / 2]$, the potential $V(x)$ may be an arbitrary strictly monotonically decreasing smooth function, while outside $[-L / 2, L / 2]$ it is fixed by Eqs. (2),(3). An example with $q<1$ is given at the end of this section.

We remark that in the generic case we have

$$
\begin{gathered}
q=3, \quad V^{\prime}(0)=V_{0}^{\prime}(0)-F_{c}=0 \\
V^{\prime \prime}(0)=V_{0}^{\prime \prime}(0)=0 \\
V_{0}^{\prime \prime \prime}(0)=-6 \mu<0 .
\end{gathered}
$$

For instance, this is so whenever $V_{0}(x)$ in Eq. (2) is an analytic function of $x$. Nevertheless, more general $q$ values are also worth studying, as our results below will demonstrate. They can be readily realized experimentally by tailoring the form of $V_{0}(x)$ accordingly. We finally note [using Eqs. (2),(3)] that $V(L / 2)-V(-L / 2)=-F L$. Assuming that Eq. (26) is a rough approximation for $V(x)$ in the entire interval $[-L / 2, L / 2]$ it follows that $-2 \mu(L / 2)^{q}-\epsilon L$ $\approx-F L$ and hence

$$
\mu \approx F_{c}(L / 2)^{1-q}
$$

Our second main assumption throughout this section is that the thermal energy $k T$ is small, in the sense that

$$
k T \ll L F_{c} .
$$

Our main goal in what follows is to determine the behavior of the diffusion coefficient $D$ for asymptotically small $\epsilon$ and $k T$.

It is instructive to extend for a moment the approximation (26) to the entire $x$ axis and to consider the corresponding dynamics (1) for $\epsilon=0$ and in the zero temperature limit $\xi(t) \equiv 0$, i.e.,

$$
\dot{x}(t)=\frac{\mu q}{\eta}|x(t)|^{q-1}
$$

A straightforward calculation then shows that in order to reach the inflection point $x=0$ from a seed $x(0)<0$, an infinite amount of time is needed if $q \geqslant 2$, while a finite time is sufficient when $q<2$ (but still $q>1$ ). On the other hand, for $x(0) \rightarrow-\infty$ a finite time suffices to reach a small neighborhood of $x=0$ for $q>2$, while this traveling time diverges for $q \leqslant 2$. Analogous results are recovered for the traveling times in the region $x>0$. As a consequence, fundamentally differ- ent kinds of behavior of the corresponding mean first passage times for $q>2$ and $q<2$ are expected also for asymptotically small but finite $k T$ (but still $\epsilon=0$ ) [40]: For $q>2$ the motion of the particle (1) is dominated by the passage through the vicinity of $x=0$, where Eq. (31) is valid, whereas for $q<2$ one expects that the passage through this region is negligible, and outside this region the influence of the noise can be ignored. A similar behavior is still expected for finite $\epsilon$-values, provided they become sufficiently small as $k T$ approaches zero, while otherwise again fundamentally different realms may be encountered. In the following subsections, a more rigorous, quantitative version of these heuristic arguments will be elaborated.

Regarding the case $q<1$ we restrict ourselves to the specific example of a critically tilted piecewise linear potential. More precisely, we consider the diffusion of particles in a step-type potential, i.e., $V(x)$ decreases proportional to the integer part of $-x / L$, with $k T$ much smaller than the potential step. This problem is a priori not simple at all, and was in fact the question that motivated the entire present investigation. However, with the general framework developed above now at hand, one readily finds from Eqs. (22) and (15) the result $D=2 D_{0} / 3$ for small $k T$, i.e., the free diffusion coefficient is reduced by the factor $2 / 3$.

\section{A. Evaluation of the diffusion coefficient}

In this subsection we derive our central results (53)-(55) for the scaling behavior of the effective diffusion coefficient.

We first focus on the evaluation of the integral $\int_{x_{0}}^{x_{0}+L} d x I_{ \pm}(x)$ appearing in Eqs. (18) and (22). To keep things simple, we temporarily focus on the index " + " and make the specific choice $x_{0}=-L / 2$, but it is clear that the final result will be valid for both indices and any $x_{0}$. Because of Eq. (31) one can replace the lower integration limit $x-L$ in Eq. (19) by $-\infty$ to a very good approximation [see also Eq. (A1) in Appendix A]. Next we evaluate a part of the integral $\int_{-L / 2}^{L / 2} d x I_{+}(x)$, namely [cf. Eq. (21)],

$$
\int_{-L / 2}^{-a} d x I_{+}(x)=\int_{-L / 2}^{-a} d x \frac{1}{D_{0}} \int_{-\infty}^{0} d z e^{[V(x)-V(x+z)] / k T},
$$

where we have introduced

$$
a:=A\left(\frac{k T}{\mu}\right)^{1 / q} \approx \frac{A L}{2}\left(\frac{2 k T}{L F_{c}}\right)^{1 / q} .
$$

Here $A$ is a dimensionless number, and in the second relation in Eq. (34) we have exploited Eq. (30). In the following, we will always assume that $A$ is very large, while $a$ is so small that Eq. (26) can be applied in the region $|x| \leqslant a$ [such a choice of $A$ and $a$ is guaranteed to be possible due to Eq. (31)]. Observing that for small $k T$ only $z$ values very close to zero contribute notably in the second integral in Eq. (33), a Taylor expansion of the integrand about $z=0$ yields after a straightforward calculation the result 


$$
\begin{aligned}
\int_{-L / 2}^{-a} d x I_{+}(x) & =\int_{-L / 2}^{-a} d x \frac{\eta}{\left|V^{\prime}(x)\right|}\left\{1+O\left(\frac{k T V^{\prime \prime}(x)}{\left[V^{\prime}(x)\right]^{2}}\right)\right\} \\
& =\int_{-L / 2}^{-a} d x \frac{\eta}{\left|V^{\prime}(x)\right|}\left\{1+O\left(A^{-q}\right)\right\} .
\end{aligned}
$$

In the last identity we have exploited the fact that $a$ in Eq. (34) belongs to the region where Eq. (26) applies if $k T$ becomes sufficiently small, and that within this region $\left|V^{\prime}(x)\right|$ will be much smaller than it is outside it. In the same way one finds that

$$
\int_{a}^{L / 2} d x I_{+}(x)=\int_{a}^{L / 2} d x \frac{\eta}{\left|V^{\prime}(x)\right|}\left\{1+O\left(A^{-q}\right)\right\} .
$$

In the remaining integral

$$
\int_{-a}^{a} d x I_{+}(x)=\int_{-a}^{a} d x \frac{1}{D_{0}} \int_{-\infty}^{0} d z e^{[V(x)-V(x+z)] / k T}
$$

again only very small $z$ values contribute significantly and one thus may exploit Eq. (26) in the entire integration domain. After changing integration variables to $\tilde{x}$ $:=x(\mu / k T)^{1 / q}, \bar{z}:=-z(\mu / k T)^{1 / q}$ and then dropping the tildes, one finds that

$$
\int_{-a}^{a} d x I_{+}(x)=\frac{\eta}{\mu^{2 / q}[k T]^{1-2 / q}} \int_{-A}^{A} d x K(x, \gamma),
$$

where [cf. Eqs. (19),(21)]

$$
\begin{gathered}
\gamma:=\epsilon /\left[\mu^{1 / q}(k T)^{1-1 / q}\right] \\
K(x, \gamma):=\int_{0}^{\infty} d z \exp \left\{-x|x|^{q-1}+(x-z)|x-z|^{q-1}-\gamma z\right\} \\
=e^{-x\left(|x|^{q-1}+\gamma\right)} \int_{-\infty}^{x} d y e^{y\left(|y|^{q-1}+\gamma\right)}
\end{gathered}
$$

Note that $K(x, \gamma)$ is a dimensionless function and that both its arguments $(x$ and $\gamma$ ) are dimensionless as well. We also remark that if we had worked with $I_{-}(x)$ instead of $I_{+}(x)$ then the results (35) and (37) would have been recovered without any modification, while on the right-hand side of Eq. (38) the integrand $K(-x, \gamma)$ would have appeared, which of course gives the same result as $K(x, \gamma)$ after integration over $x$.

Upon adding up the three contributions (35), (36), (38) one sees that for $q>2$ the last one dominates for any small but fixed choice of $a$ in Eq. (34) as $k T$ becomes small. In particular, the latter contribution (38) converges for $k T \rightarrow 0$, which is basically a consequence of the fact that the mean first passage time to infinity is finite for $q>2$. Recalling that the final result does not depend on the specific choice $x_{0}$ $=-L / 2$ and the index " + ", we can conclude that [31]

$$
\int_{x_{0}}^{x_{0}+L} d x I_{ \pm}(x)=\frac{\eta G_{1}(\gamma)}{\mu^{2 / q}[k T]^{1-2 / q}} \quad \text { for } q>2
$$

$$
G_{1}(\gamma):=\int_{-\infty}^{\infty} d x K( \pm x, \gamma)
$$

up to a relative error that approaches zero as $k T$ decreases and provided that $\epsilon$ also decreases such that the dimensionless number $\gamma$ in Eq. (39) remains constant. Note that Eq. (41) has the form of a scaling law with a completely universal, dimensionless scaling function $G_{1}$ for any given value of the "critical exponent" $q$. The equivalence of both signs in Eq. (42) is obvious, but we have kept both of them in order to indicate the effect of the two possible signs in Eq. (41).

In the opposite case $q<2$ things are more complicated since later we will be interested not only in asymptotically small $k T$ and $\epsilon$ with $\gamma$ from Eq. (39) kept fixed, but also in the case of negative $\epsilon$ values such that the corresponding negative $\gamma$ values diverge logarithmically as $k T$ approaches zero. To this end, we henceforth set

$$
A:=\left(L^{q} \mu / k T\right)^{2 / q} .
$$

In this way, $A \gg|\gamma|$ as $k T \rightarrow 0$ and $a$ form (34) tends to zero [other choices than in Eq. (43) with the same properties would also be possible]. It follows that the contributions of order $A^{-q}$ in Eqs. (35) and (36) can be neglected for asymptotically small $k T$. By closer inspection one further can deduce that in the remaining integrals (35) and (36) a $\gamma$ value that diverges at most logarithmically with $k T$ has an asymptotically negligible effect as well, i.e., we can formally set $\epsilon=0$ in those integrals. The remaining integrals in Eqs. (35) and (36) converge if one formally lets $a$ tend to zero, implying the asymptotically exact approximation

$$
\begin{aligned}
\int_{x_{0}}^{x_{0}+L} d x I_{ \pm}(x)= & \frac{\eta \hat{G}_{1}(\gamma)}{\mu^{2 / q}[k T]^{1-2 / q}} \\
& +\int_{x_{0}}^{x_{0}+L} \frac{\eta d x}{F_{c}-V_{0}^{\prime}(x)} \text { for } 1<q<2,
\end{aligned}
$$

where

$$
\hat{G}_{1}(\gamma):=\int_{-A}^{A} d x K( \pm x, \gamma)
$$

Note the implicit $k T$ dependence of $\hat{G}_{1}$ via Eq. (43). In spite of this dependence, one finds that the first term on the righthand side of Eq. (44) is negligible in comparison with the second for asymptotically small $k T$ with the possible exception of very large, negative $\gamma$ values. In the latter case, we may evaluate Eq. (45) by means of a saddle point approximation with the result $\hat{G}_{1}(\gamma) \simeq S(\gamma)$, where

$$
\begin{aligned}
S(\gamma):= & \Theta(-\gamma) \frac{2 \pi|\gamma / q|^{(2-q) /(q-1)}}{q(q-1)} \exp \{2(q \\
& \left.-1)|\gamma / q|^{q /(q-1)}\right\}
\end{aligned}
$$

The Heaviside step function $\Theta(-\gamma)$ has been introduced in order to make $S(\gamma)$ well defined for arbitrary $\gamma$. Closer in- 
spection shows that the difference $\hat{G}_{1}(\gamma)-S(\gamma)$ is for all values of $\gamma$ negligible in comparison with the second term on the right-hand side of Eq. (44). In other words, we obtain the result

$$
\begin{aligned}
\int_{x_{0}}^{x_{0}+L} d x I_{ \pm}(x)= & \frac{\eta S(\gamma)}{\mu^{2 / q}[k T]^{1-2 / q}} \\
& +\int_{x_{0}}^{x_{0}+L} \frac{\eta d x}{F_{c}-V_{0}^{\prime}(x)} \text { for } 1<q<2 .
\end{aligned}
$$

As already noticed, for positive or moderately negative $\gamma$ values the second term on the right-hand side dominates. Since the quantity (47) is basically equivalent to the mean first passage time $T_{1}\left(x_{0} \rightarrow x_{0}+L\right)$ [cf. Eq. (A3)] this approximate $k T$ independence in Eq. (47) is in agreement with our heuristic discussion above [see also below Eq. (31)]. On the other hand, for sufficiently large negative $\gamma$ values, the first term on the right-hand side in Eq. (47) takes over, reproducing the expected Arrhenius-type behavior (46) for the escape time over a potential barrier [32]. Finally, we note that also in the case $q>2$ a saddle point approximation for large negative $\gamma$ values in Eq. (42) leads to the very same result $S(\gamma)$ as in Eq. (46). that

The above results imply for the current $\langle\dot{x}\rangle$ in Eq. (18)

$$
\begin{gathered}
\langle\dot{x}\rangle=\frac{D_{0}}{L}\left(\frac{L^{q} \mu}{k T}\right)^{2 / q} \frac{1}{G_{1}(\gamma)} \text { for } q>2, \\
\langle\dot{x}\rangle=\frac{D_{0}}{L} \frac{L^{q} \mu}{k T} \frac{1}{\left(\frac{k T}{L^{q} \mu}\right)^{2 / q-1} S(\gamma)+\int_{x_{0}}^{x_{0}+L} \frac{d x \mu L^{q-2}}{F_{c}-V_{0}^{\prime}(x)}}
\end{gathered}
$$$$
\text { for } 1<q<2 \text {. }
$$

The "crossover" case $q=2$ requires a separate treatment that is relegated to the Appendix B.

For the evaluation of the numerator in Eq. (22) one proceeds in exactly the same way as for the denominator, and we only report here the final results,

$$
\int_{x_{0}}^{x_{0}+L} d x I_{ \pm}(x) I_{+}(x) I_{-}(x)=\frac{\eta^{3} G_{3}(\gamma)}{\mu^{4 / q}[k T]^{3-4 / q}} \quad \text { for } q>4 / 3,
$$

$$
\begin{aligned}
G_{3}(\gamma):=\int_{-\infty}^{\infty} d x K( \pm x, \gamma) K(x, \gamma) K(-x, \gamma), \\
\int_{x_{0}}^{x_{0}+L} d x I_{ \pm}(x) I_{+}(x) I_{-}(x)=\frac{\eta^{3}[S(\gamma)]^{2} / 2}{\mu^{4 / q}[k T]^{3-4 / q}} \\
+\int_{x_{0}}^{x_{0}+L} \frac{\eta^{3} d x}{\left[F_{c}-V_{0}^{\prime}(x)\right]^{3}} \\
\text { for } 1<q<4 / 3 .
\end{aligned}
$$

As in Eq. (42), we have kept in Eq. (51) both signs, though their equivalence is obvious. Moreover, for $q>4 / 3$ a saddle point approximation for large negative $\gamma$ values in Eq. (51) again leads to the same expression $[S(\gamma)]^{2} / 2$ as appearing in Eq. (52). For the "crossover" case $q=4 / 3$, see Appendix B.

The implications of the above results for the diffusion in Eq. (22) are

$$
\begin{gathered}
D=D_{0}\left(\frac{L^{q} \mu}{k T}\right)^{2 / q} \frac{G_{3}(\gamma)}{\left[G_{1}(\gamma)\right]^{3}} \text { for } q>2, \\
D=D_{0}\left(\frac{L^{q} \mu}{k T}\right)^{3-4 / q} \\
\times \frac{G_{3}(\gamma)}{\left[\left(\frac{k T}{L^{q} \mu}\right)^{2 / q-1} S(\gamma)+\int_{x_{0}}^{x_{0}+L} \frac{d x \mu L^{q-2}}{F_{c}-V_{0}^{\prime}(x)}\right]^{3}} \\
D_{0} \frac{\left(\frac{k T}{L^{q} \mu}\right)^{4 / q-3}[S(\gamma)]^{2}}{2}+\int_{x_{0}}^{x_{0}+L} \frac{d x \mu^{3} L^{3 q-4}}{\left[F_{c}-V_{0}^{\prime}(x)\right]^{3}} \\
{\left[\left(\frac{k T}{L^{q} \mu}\right)^{2 / q-1} S(\gamma)+\int_{x_{0}}^{x_{0}+L} \frac{d x \mu L^{q-2}}{F_{c}-V_{0}^{\prime}(x)}\right]^{3}}
\end{gathered}
$$

We recall that $G_{1}(\gamma), S(\gamma)$, and $G_{3}(\gamma)$ from Eqs. (42), (46), and (51), respectively, are dimensionless scaling functions of their dimensionless argument (39) that are completely universal for any given $q$ value. Similarly, the fraction $L^{q} \mu / k T$ as well as all the integrals appearing in Eqs. (49)-(55) are dimensionless numbers. Under the approximative assumption that Eq. (26) is valid in the entire interval $[-L / 2, L / 2]$ one obtains for those integrals the result

$$
\begin{gathered}
\int_{x_{0}}^{x_{0}+L} \frac{d x \mu L^{q-2}}{F_{c}-V_{0}^{\prime}(x)}=\frac{2^{q-1}}{q(2-q)}, \\
\int_{x_{0}}^{x_{0}+L} \frac{d x \mu^{3} L^{3 q-4}}{\left[F_{c}-V_{0}^{\prime}(x)\right]^{3}}=\frac{8^{q-1}}{q^{3}(4-3 q)} .
\end{gathered}
$$

The special "crossover" values $q=2$ and $q=4 / 3$ are addressed in Appendix B. Basically, these $q$-values continuously (but not smoothly) match together the results (53)(55), involving certain logarithmic corrections similarly as it is the case for crossover exponents in the context of critical phenomena.

\section{B. Discussion and examples}

In this subsection we discuss Eqs. (53)-(55).

As far as the result (53) for $q>2$ is concerned, the most remarkable feature is the divergence of $D / D_{0}$ when $k T$ tends to zero for any fixed $\gamma$ value. In other words, we recover $a$ giant enhancement of thermal diffusion (cf. Fig. 1). Specifically, for $q=3$, i.e., the most important case in practice [cf. Eq. (29)], the scaling function $G(\gamma):=G_{3}(\gamma) / G_{1}^{3}(\gamma)$ appear- 


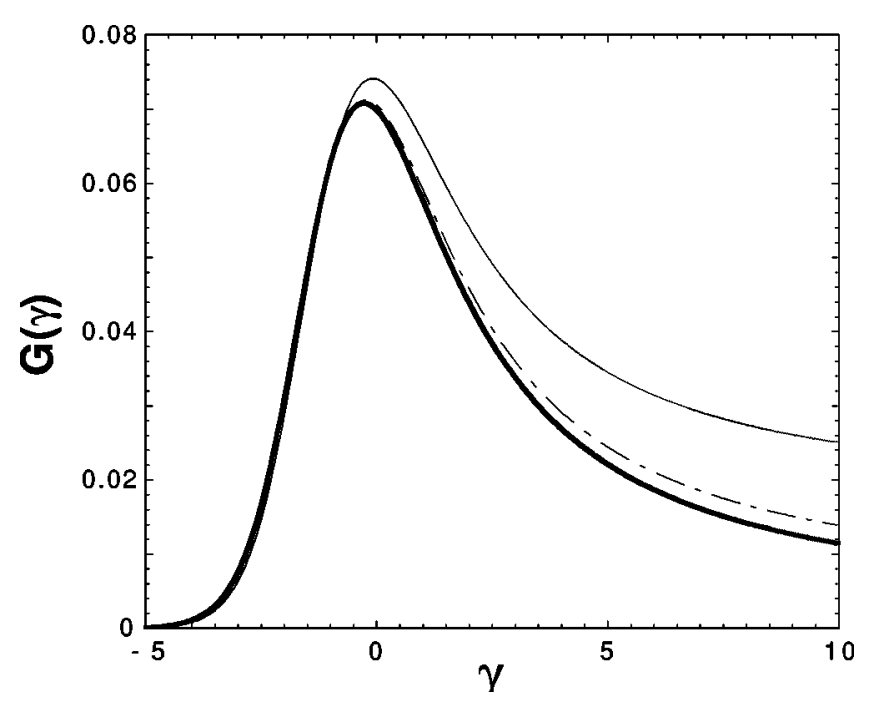

FIG. 2. Bold solid line: Dimensionless scaling function $G(\gamma)$ $:=G_{3}(\gamma) / G_{1}^{3}(\gamma)$ in Eq. (53) for $q=3$ versus its dimensionless argument $\gamma$ from Eq. (39) by numerically evaluating Eqs. (42),(51). Full and dashed-dotted lines: Same as the respective lines in Fig. 1, but now plotted in the form $\left(D / D_{0}\right)\left(k T / L^{q} \mu\right)^{2 / q}[\mathrm{cf}$. Eq. (53)] versus $\gamma$ [cf. Eqs. (25),(39)] with $q=3$ [cf. Eqs. (23),(29)].

ing in (53) is depicted in Fig. 2. From this plot it follows that the scaling form (53) is rather well satisfied already for moderately small $k T$ values and that the enhancement of diffusion is most pronounced for $\gamma$ values in Eq. (39) of the order unity. A similar behavior is recovered for any other $q>2$. In other words, for $q>2$ the diffusion coefficient as a function of $F$ exhibits a pronounced peak at $F=F_{c}$ with a height of the order $D_{0}\left(L^{q} \mu / k T\right)^{2 / q}$ and a width $\Delta F=\Delta \epsilon$ of the order $\mu^{1 / q}[k T]^{1-1 / q}\left[\mathrm{cf}\right.$. (39)]. Exploiting that $D_{0} \propto T$ [cf. (7)] we have, in particular, that

$$
\begin{gathered}
D\left(\gamma_{\max }\right) \sim D_{0}^{1-2 / q} \propto T^{1-2 / q}, \\
\gamma_{\max } \simeq 0 .
\end{gathered}
$$

Further, we note that for large negative $\gamma$ values one can exploit in Eq. (53) the saddle point approximation (46) for $G_{1}(\gamma)$ and the corresponding approximation $[S(\gamma)]^{2} / 2$ for $G_{3}(\gamma)$ as discussed below (52).

Returning to the height of the peak in the special case $q$ $=3$ the numerics from Fig. 2 in combination with Eq. (53) implies that

$$
D \simeq 0.0696 D_{0}\left(\frac{L^{3} \mu}{k T}\right)^{2 / 3} \text { for } q=3, \quad F=F_{c} .
$$

We remark that in the present special case $q=3$, the integrations in Eq. (42) can be exchanged and the integral over $x$ performed, with the result

$$
G_{1}(\gamma)=\int_{0}^{\infty} d x e^{\left(-\gamma x^{2}-x^{6} / 4\right)}
$$

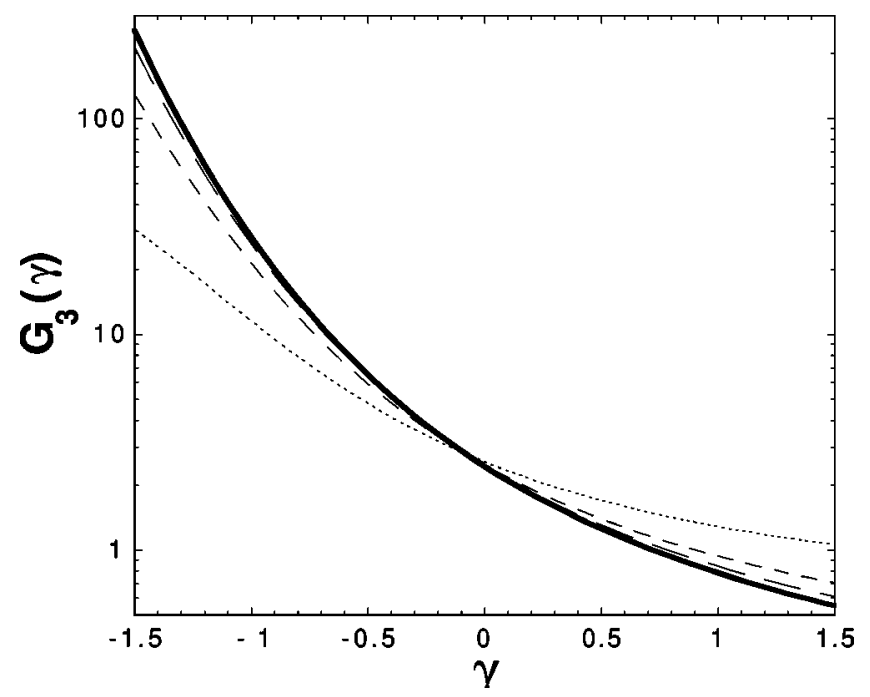

FIG. 3. Bold solid line: Dimensionless scaling function $G_{3}(\gamma)$ in Eq. (54) for $q=3 / 2$ versus its dimensionless argument $\gamma$ from Eq. (39) by numerically evaluating Eq. (51). Other lines: The quantity $\left(D / D_{0}\right)\left(k T / L^{q} \mu\right)^{3-4 / q}\left[2^{q-1} / q(2-q)\right]^{3}$ [cf. Eqs. (54),(56), and main text] with $D$ from Eq. (22) versus $\gamma$ [cf. Eq. (39)] for a potential $V(x)$ that is given by Eq. (26) for all $x \in[-L / 2, L / 2]$ while outside $[-L / 2, L / 2]$ it is fixed by Eqs. (2),(3). Parameter values in dimensionless units: $L=2, \mu=1, \eta=1, k T=D_{0}=10^{-3}$ (dotted), $k T$ $=D_{0}=10^{-5}$ (short dashes), $k T=D_{0}=10^{-7}$ (long dashes).

Similarly, the integral over $x$ can also be performed in Eq. (51). Further analytical simplifications are only possible for $\gamma=0$. In this case, Eq. (61) can be expressed as

$$
G_{1}(0)=2^{1 / 3} \sqrt{\pi} \Gamma(1 / 6) / 3^{3 / 2} \simeq 2.39,
$$

where $\Gamma(z):=\int_{0}^{\infty} d t t^{z-1} e^{-t}$ is the Gamma function with $\Gamma(1 / 6) \simeq 5.57$. Due to a remarkable mathematical identity by Sigeti and Horsthemke [12] one further finds for the scaling function in Eq. (51) the result

$$
G_{3}(0)=\left[G_{1}(0)\right]^{2} / 6
$$

By means of this result one analytically recovers Eq. (60). Note also the difference between the relation (63) for $\gamma=0$ and the asymptotic behavior $G_{3}(\gamma)=\left[G_{1}(\gamma)\right]^{2} / 2$ for large negative $\gamma$ values as discussed below Eq. (52).

Next, we turn to the discussion of the result (54) for $4 / 3$ $<q<2$. The salient difference in comparison with Eq. (53) is a competition between the two terms in the denominator on the right-hand side of Eq. (54). For any fixed $\gamma$ value, the first term is negligible when $k T$ becomes sufficiently small. Thus $D / D_{0}$ increases proportional to $[k T]^{4 / q-3}$, i.e., we find again a giant enhancement of thermal diffusion. More subtle is the behavior of Eq. (54) as a function of $\gamma$ for a small but fixed $k T$ value. For arbitrary positive as well as for moderately negative $\gamma$ values it is still the second term in the denominator that dominates and thus the $\gamma$ dependence of $D$ is governed by $G_{3}(\gamma)$. These predictions are confirmed by comparison with a direct evaluation of the exact formula (22), see Fig. 3 for an example. In contrast to the case $q$ $=3$ (cf. Fig. 2) the asymptotic scaling form (54) with $S(\gamma)$ 
$\rightarrow 0$ is approached only for rather small $k T$ values in Fig. 3 . On the other hand, for large negative $\gamma$ values we can make use of the saddle point approximation $[S(\gamma)]^{2} / 2$ for $G_{3}(\gamma)$ as discussed below Eq. (52). Since $S(\gamma)$ from Eq. (46) increases very fast with decreasing $\gamma$, the right-hand side of Eq. (54) increases very fast as long as $G_{3}(\gamma)$ governs the $\gamma$ dependence. However, again due to this fast increase, the first summand in the denominator starts to compete with the second summand and ultimately takes over, leading to a decrease of $D$ proportional to $1 / S(\gamma)$. Thus a peak appears at a (negative) $\gamma$ value for which both terms in the denominator are of the same order of magnitude. The detailed quantitative calculation is straightforward and leads to the result

$$
D\left(\gamma_{\max }\right)=D_{0} \frac{2}{27} \frac{L^{q} \mu}{k T}\left[\int_{x_{0}}^{x_{0}+L} \frac{d x \mu L^{q-2}}{F_{c}-V_{0}^{\prime}(x)}\right]^{-1}
$$

where $\gamma_{\max }$ is defined via the transcendental equation

$$
\left(\frac{k T}{L^{q} \mu}\right)^{2 / q-1} S\left(\gamma_{\max }\right)=2 \int_{x_{0}}^{x_{0}+L} \frac{d x \mu L^{q-2}}{F_{c}-V_{0}^{\prime}(x)} .
$$

For small $k T$ one thus obtains with Eq. (46) the leading order solution

$$
\gamma_{\max } \simeq-q\left[\frac{2-q}{2 q(q-1)} \ln \left(\frac{L^{q} \mu}{k T}\right)\right]^{1-1 / q}<0 .
$$

The width $\Delta \gamma$ of the peak is found to be of the order

$$
\Delta \gamma \simeq\left(\frac{q}{-\gamma_{\max }}\right)^{1 /(q-1)}
$$

First of all, we note the logarithmic $k T$ dependence in Eq. (66). In other words, the peak region is self-consistently described by our calculations, see above Eq. (43). Second, taking into account Eq. (7) we see that the maximal effective diffusion coefficient in Eq. (64) is in fact independent of $k T$. In other words, the maximal enhancement of diffusion is even stronger than for $q>2$, see Eq. (58). Under the approximative assumption that Eq. (26) is valid in the entire interval $[-L / 2, L / 2]$ one obtains with Eq. (56) the explicit result [31]

$$
D\left(\gamma_{\max }\right)=\frac{2^{2-q} q(2-q)}{27} \frac{L^{q} \mu}{\eta}
$$

independent of $k T$. These asymptotic predictions for $k T \rightarrow 0$ are nicely confirmed already for moderately small $k T$ values by comparison with a direct numerical evaluation of the exact formula (22) in Fig. 4. Note that while the maximizing $\gamma$ value in Eq. (66) tends to $-\infty$ as $k T \rightarrow 0$, the corresponding tilt $\epsilon=F-F_{c}$ in Eq. (39) tends to zero. While $k T$ may become arbitrarily small, the case $k T=0$ is not included in our above calculations, basically since this limit is singular in Eq. (10) and thus in Eq. (22). The basic physical reason for this singularity is the fact that the passage time through the interval $[-L / 2, L / 2]$ remains finite for any finite $k T$ but becomes infinite for $k T=0$.

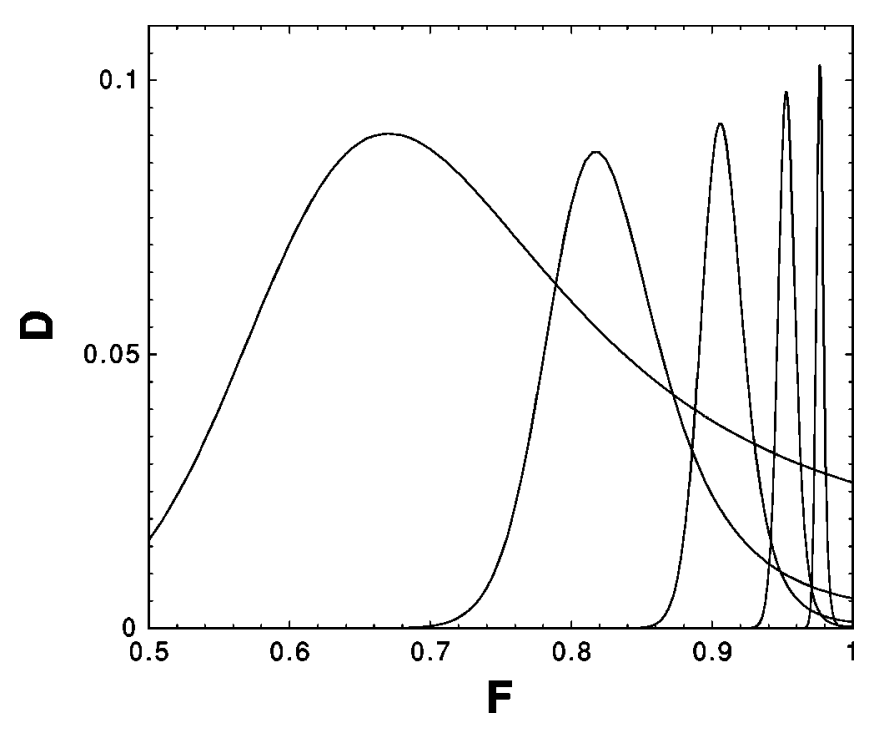

FIG. 4. Diffusion coefficient (22) versus the tilt $F$ for a potential $V(x)$ defined via Eqs. (25),(26) with $q=3 / 2$ for all $x$ $\in[-L / 2, L / 2]$ while outside $[-L / 2, L / 2]$ it is fixed by Eqs. (2),(3). Using dimensionless units, the parameter values are $\eta=1, L=2, \mu$ $=1, F_{c}=1$. The five curves with increasingly sharper peaks correspond to the following five values of $k T=D_{0}: 3 \times 10^{-2}, 10^{-2}$, $10^{-3}, 10^{-4}$, and $10^{-5}$. The theoretically predicted peak height for asymptotically small $k T$ from Eq. (68) is $0 . \overline{1}$.

Finally, we turn to the discussion of the result (55) for 1 $<q<4 / 3$. For positive and moderately negative $\gamma$ values the two integrals on the right-hand side of Eq. (55) dominate and thus the diffusion coefficient is essentially constant. Consequently, the diffusion $D$ is proportional to the bare value $D_{0}$ and in the limit $V_{0}^{\prime}(x) \rightarrow F_{c}+\epsilon$, corresponding to $q \rightarrow 1$, the correct behavior (7) is also recovered. On the other hand, with increasingly negative $\gamma$ values, the first term in the numerator in Eq. (55) takes over, while in the denominator the integral is still dominating. In other words, we essentially recover the same behavior as in Eq. (54). Especially, a peak of the form (64)-(68) arises for any $1<q<2$, cf. Fig. 4.

In the case $q>2$ the approximation by Constantini and Marchesoni in Eq. (24) can be evaluated by means of Eqs. (18),(31),(39), and (41), leading to

$$
D=D_{0}\left(\frac{L^{q} \mu}{k T}\right)^{1 / q} \frac{\left[-G_{1}^{\prime}(\gamma)\right]}{\left[G_{1}(\gamma)\right]^{2}} \text { for } q>2 .
$$

Comparison with the exact asymptotics (53) shows that this approximation does not capture the correct scaling functions and exponents. A similar disagreement is obtained for $1<q$ $<2$. On the other hand, one readily finds that the main qualitative features are correctly reproduced in all cases.

\section{Basic physical mechanism}

The basic physical mechanism responsible for the enhancement of the thermal diffusion may be understood along the following heuristic argument. As discussed below Eq. (32), for $q>2, \epsilon=0$, and small $k T$, the noisy dynamics (1) is dominated by the passage through the "dynamical bottle- 
neck" located at the inflection point $x=0$, cf. Eq. (26). Since $\epsilon=0$, a very small perturbation due to thermal noise is already sufficient to kick the particle across the inflection point $x=0$. This small variation in comparison with an unperturbed particle is subsequently greatly enhanced by the further dynamical evolution. The result is a huge dispersion for a statistical ensemble of particles subjected to different realizations of the noise. It is quite suggestive that the same basic mechanism subsists also for nonvanishing $\epsilon$ values at least as long as they are so small that the corresponding $\gamma$ values in Eq. (39) remain finite when $k T$ approaches zero.

Due to the same mechanism, one expects a very strong change of the particle current $\langle\dot{x}\rangle$ upon small changes of the tilt $F$ near the critical value $F_{c}$. In other words, a relation like in Eq. (24) is expected to be correct at least qualitatively. However, it is clear that this heuristic argument can be applied only for $q>2$, because only then the "bottleneck" near $x=0$ dominates the dynamics [cf. the discussion below Eq. (32)]. Since we have found an enhanced diffusion also for $\epsilon=0$ and $4 / 3 \leqslant q \leqslant 2$ we have to conclude that such a simple argument only captures a part of the essential physics and that a relation of the form (24) cannot be strictly correct [cf. Fig. 1 and the discussion below Eq. (69)].

Interestingly enough, a similar heuristic explanation in terms of dynamically enhanced thermal fluctuations seems again applicable for $2>q>1$ near the peak in the diffusion coefficient described by Eqs. (64)-(68): As discussed above Eq. (64), in this peak region both summands in the denominator of Eq. (54) are comparable, reflecting the fact that the passage time through a very small neighborhood of $x=0$ is comparable to the traveling time through the remainder of $[-L / 2, L / 2]$. On the other hand, the large negative $\gamma$ value in Eq. (66) and the concomitant Arrhenius-type form of Eq. (46) indicate that the former time scale is governed by a thermally activated escape process across a potential barrier. On this basis, it is quite plausible that the dynamical enhancement of these thermally induced (rare) escape events will be maximal when both time scales are comparable.

The discussion below Eq. (32) implies that for $q>2$ the mean first passage time $T_{1}\left(x_{0} \rightarrow x_{0}+L\right)$ is dominated by a small neighborhood of the inflection point $x=0$, while for $2>q>1$ the region outside this small neighborhood is no longer negligible. From our results in Eqs. (50),(52) we can conclude that a similar crossover occurs for the first passage time dispersion $\Delta T_{2}\left(x_{0} \rightarrow x_{0}+L\right)$ at $q=4 / 3$. In view of our central relations (14) and (15) the need to distinguish between two $q$ regimes for the current $\langle\dot{x}\rangle$ in Eqs. (48),(49) and three $q$ regimes for the diffusion $D$ in Eqs. (53)-(55) is then immediately clear.

We finally note that a suitable comparison between the directed and the diffusive transport is provided by the dimensionless number [41]

$$
Q:=\frac{2 D}{L\langle\dot{x}\rangle}=\frac{\Delta T_{2}\left(x_{0} \rightarrow x_{0}+L\right)}{\left[T_{1}\left(x_{0} \rightarrow x_{0}+L\right)\right]^{2}}=\lim _{t \rightarrow \infty} \frac{\left\langle x^{2}(t)\right\rangle-\langle x(t)\rangle^{2}}{L\langle x(t)\rangle},
$$

where the second equality follows from Eqs. (14),(15) and the third from Eqs. (5),(6). For large positive $\gamma$ one finds that

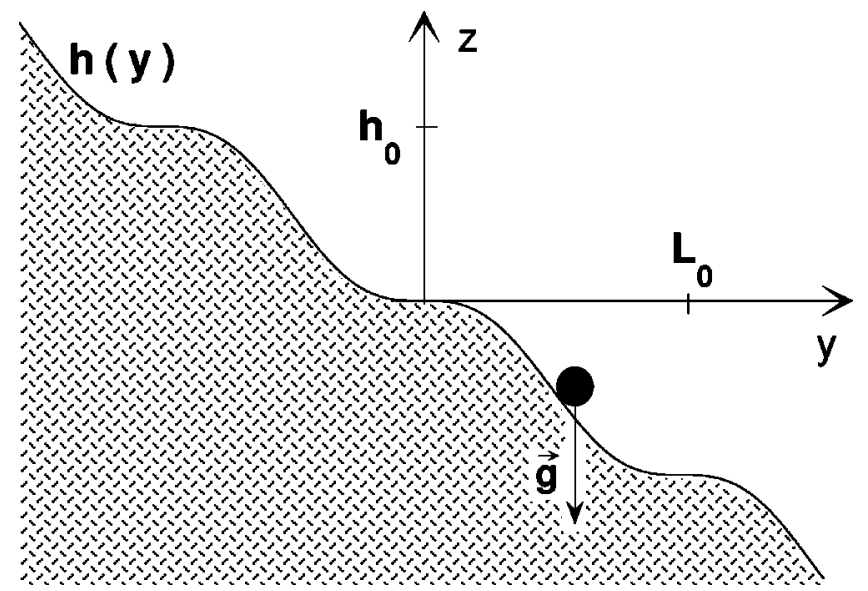

FIG. 5. A spherical Brownian particle in a liquid, rolling down a critically tilted, periodic surface $h(y)$ under the action of gravitation, see also Eq. (71).

$Q$ becomes very small, while for large negative $\gamma$ one finds that $Q$ approaches unity. For $q=3$ and $\gamma=0 \simeq \gamma_{\max }$ [cf. Eq. (59)] the relation (63) implies $Q=1 / 3$, whereas for $2>q$ $>1$ and $\gamma=\gamma_{\max }$ [cf. Eq. (66)] one finds that $Q=2 / 9$. In other words, in the most interesting regime close to the maximum of the diffusion coefficient the particle dispersion $\left\langle x^{2}(t)\right\rangle-\langle x(t)\rangle^{2}$ is comparable to $L$ times the mean displacement $\langle x(t)\rangle$ and similarly the first passage time dispersion is comparable to the square of the mean first passage time.

\section{MACROSCOPIC THERMAL DIFFUSION}

In this section we consider the thermally induced diffusion of a real mechanical particle of spherical shape that moves in a liquid under the action of gravitation along the rigid surface of a critically tilted periodic geometrical profile, see Fig. 5.

The position of the particle is described by its coordinate $y$ along some horizontal axis and its vertical position $z$. Gravitation is pointing in the negative $z$ direction and the motion is constrained by a rigid surface according to $z$ $\geqslant h(y)$, where $h(y)$ has the shape of a periodic profile with period $L_{0}$ that is critically tilted "to the right," i.e.,

$$
h\left(y+L_{0}\right)=h(y)-h_{0},
$$

with $h_{0}>0$, see Fig. 5. The motion along the third spatial direction (perpendicular to both $y$ and $z$ ) decouples from the motion in the $y-z$ plane and can, therefore, be ignored. Without loss of generality we assume that $h(y)$ has an inflection point at $y=0$ and thus satisfies for small $y$ a relation analogous to Eq. (26) with $\epsilon=0$, i.e.,

$$
h(y)=-\mu_{0} y|y|^{q-1} .
$$

Physically, it is quite plausible that for asymptotically small $k T$, the constraint $z \geqslant h(y)$ can henceforth be replaced by $z=h(y)$ without changing the dynamics along the $y$ direction. A more detailed mathematical justification of this step is possible but not further elaborated at this place. At 
every given position $[y, h(y)]$, the thermal noise acting on the particle consists of a perpendicular and a tangential component relative to the geometrical profile $h(y)$. Under the assumption that the thermal fluctuations can be modeled by Gaussian white noise, these two components are statistically independent at any given time and position. Furthermore, the perpendicular component has no effect because of the constraint $z=h(y)$. In order to achieve an effective onedimensional description of the form (1) the appropriate approach is thus to work with the path-length $x$ along the geometrical profile as our generalized coordinate. The infinitesimal line element $d x$ is then specified by the obvious relation $d x^{2}=d y^{2}+d h^{2}$ and the sign convention in the relation $d x= \pm\left(d y^{2}+d h^{2}\right)^{1 / 2}$. Without loss of generality we choose the sign convention such that $d x=d y\left(1+\left[h^{\prime}(y)\right]^{2}\right)^{1 / 2}$ and the $x$ origin such that

$$
x(y)=\int_{0}^{y} d \hat{y}\left(1+\left[h^{\prime}(\hat{y})\right]^{2}\right)^{1 / 2} .
$$

The potential energy of the particle with density $\rho$ and radius $r$ due to the effects of gravitation and the buoyancy of the surrounding liquid with density $\rho_{l}$ is given in terms of the generalized coordinate $x$ by

$$
V(x)=F_{g} h(y(x)), \quad F_{g}:=(4 \pi / 3) r^{3}\left(\rho-\rho_{l}\right) g,
$$

where $y(x)$ is the inverse of $x(y)$ (which obviously exists) and where $g$ is the acceleration due to gravity. Observing that $h^{\prime}(0)=0$ in Eq. (73) it follows that $V(x)$ is a critically tilted periodic potential that satisfies Eq. (26) for small $x$ with

$$
\epsilon=0, \quad \mu=F_{g} \mu_{0} .
$$

With Eqs. (2), (3), (71), (73), and (74) it follows that

$$
\begin{gathered}
F_{c} L=V(x)-V(x+L)=F_{g} h_{0}, \\
L=\int_{0}^{L_{0}} d \hat{y}\left(1+\left[h^{\prime}(\hat{y})\right]^{2}\right)^{1 / 2} .
\end{gathered}
$$

We assume that the frictional force acting on the particle under consideration is of the Stokes form $\eta \dot{x}(t)$ with friction coefficient

$$
\eta=6 \pi \nu_{\text {eff }} r,
$$

where $\nu_{\text {eff }}$ is the effective viscosity of the surrounding liquid. For a spherical particle that does not rotate and that is surrounded by an unbounded reservoir of liquid, $\nu_{\text {eff }}$ is given by the bare viscosity of this liquid. A rigorous quantitative theory describing the thermal motion [note that $\eta$ also appears in Eq. (4)] of the actual setup we have in mind should include the effects of rotational degrees of freedom and the quite intricate boundary effects, both mechanical and hydrodynamical [42]. Here, we shall adopt the simplifying assumption [43] that all these effects are approximately captured by an appropriately renormalized viscosity $\nu_{\text {eff }}$. Further, we assume that all kinds of fluctuations within the liquid (density, temperature, etc.) are approximately captured by the thermal noise $\xi(t)$ in Eq. (1). Finally, we assume incompressibility of the liquid (and the particle) such that the densities $\rho$ and $\rho_{l}$ are constant, and we exclude temperature gradients.

Finally, we should justify the assumption that the inertia term $m \ddot{x}(t)$, missing on the left-hand side of Eq. (1), is indeed negligible, at least in those aspects of the dynamics that are responsible for the asymptotic behavior in Eqs. (53)(55). In practice, this is a very delicate question and a truly satisfactory answer seems only possible by means of a comparison with numerical simulations that fully take into account these small but finite inertia effects. Intuitively, we expect that there is a range of small but still macroscopic particle sizes for which the overdamped description (1) would be admissible. A closely related question concerns the omission of memory effects both in the noise and the dissipation, which can be justified by closer inspection under the condition that the particle density $\rho$ is much larger than the liquid density $\rho_{l}$.

In the important case $q=3$, and with

$$
\begin{gathered}
g \simeq 981 \mathrm{~cm} / \mathrm{s}^{2}, \\
\hat{\nu}:=10^{-2} \mathrm{~g} / \mathrm{cm} \mathrm{s} \simeq \nu_{\text {water }}, \\
\hat{\rho}:=10 \mathrm{~g} / \mathrm{cm}^{3} \quad\left[\rho_{\text {iron }} \simeq 7.9 \mathrm{~g} / \mathrm{cm}^{3}\right], \\
\hat{r}:=0.1 \mathrm{~cm}, \\
\hat{h}_{0}:=1.5 \mathrm{~cm},
\end{gathered}
$$

$\hat{T}:=293^{\circ} \mathrm{K} \quad$ [room temperature],

the free diffusion coefficient (7) can be written, using Eq. (78), as

$$
D_{0} \simeq 2.14 \times 10^{-12} \frac{T}{\hat{T}} \frac{\hat{\nu}}{\nu_{\mathrm{eff}}} \frac{\hat{r}}{r} \frac{\mathrm{cm}^{2}}{\mathrm{~s}} .
$$

Further, one obtains from Eq. (60) for the diffusion coefficient at the critical tilt the formula

$$
\begin{aligned}
& D \simeq 4.99 \times 10^{-3} \frac{\hat{\nu}}{\nu_{\text {eff }}} \frac{r}{\hat{r}}\left(\frac{T}{\hat{T}}\right)^{1 / 3}\left(\frac{\rho-\rho_{l}}{\hat{\rho}} \frac{L^{3} \mu_{0}}{4 \hat{h}_{0}}\right)^{2 / 3} \frac{\mathrm{cm}^{2}}{\mathrm{~s}} \\
& \text { for } q=3 .
\end{aligned}
$$

In the actual experimental realization, which is presently under construction in the labs of one of the present authors (H.L.), each fraction appearing in Eqs. (85) and (86) is of the order of unity [44]. In particular, for typical shapes $h(x)$ one finds that $L^{q} \mu_{0} / 2^{q-1} h_{0}$ is a dimensionless number of the order of unity [cf. Eqs. (30),(75),(76),(77)], independently of the actual value of $L$. It follows that the diffusion coefficient in the critically tilted periodic potential (86) is enhanced in comparison with the free thermal diffusion coefficient (85) by about nine orders of magnitude, so that it may well reach macroscopically observable values. The width of the peak of the diffusion coefficient with respect to variations of the po- 
tential tilt has been estimated above Eq. (58), namely, $\Delta F$ $\simeq \mu^{1 / q}[k T]^{1-1 / q}$. This width of the peak may be used to estimate the experimentally admissible deviation $\Delta h_{0}$ from the exact "critical" $h_{0}$ value in Eq. (71). Due to Eq. (76) we see that $\Delta F=\Delta h_{0} F_{g} / L$, yielding the following theoretical estimate for $\Delta h_{0}$ :

$$
\frac{\Delta h_{0}}{\hat{h}_{0}} \simeq 1.2 \times 10^{-10}\left(\frac{L^{3} \mu_{0}}{4 \hat{h}_{0}}\right)^{1 / 3}\left(\frac{T}{\hat{T}} \frac{\hat{\rho}}{\rho-\rho_{l}}\right)^{2 / 3} \frac{\hat{r}^{2}}{r^{2}} .
$$

Since the fractions on the right-hand side are again of the order of unity, this theoretically required precision is thus extremely high.

As discussed in the previous section, the largest enhancement of thermal diffusion is expected for $2>q>1$. From Eq. (64) we then find for the maximal diffusion coefficient the result

$$
\begin{aligned}
D \simeq 2.42 \times 10^{2} q(2-q) \frac{\hat{\nu}}{\nu_{\mathrm{eff}}} \frac{r^{2}}{\hat{r}^{2}} \frac{\rho-\rho_{l}}{\hat{\rho}} \frac{L^{q} \mu_{0}}{2^{q-1} \hat{h}_{0}} \\
\quad \times \frac{\frac{2^{q-1}}{q(2-q)}}{\int_{x_{0}}^{x_{0}+L} \frac{d x \mu L^{q-2}}{F_{c}-V_{0}^{\prime}(x)}} \frac{\mathrm{cm}^{2}}{\mathrm{~s}} \text { for } 2>q>1,
\end{aligned}
$$

see also Eqs. (56) and (68). Again, all the fractions in the right-hand side are of order unity. In other words, if the fabrication of such a specially tailored profile $h(y)$ with $2>q$ $>1$ is possible with the necessary precision, then the enhancement of thermal diffusion can be further improved by another five orders of magnitude as compared to the case $q$ $=3$. For the required precision to hit the correct $h_{0}$ value in Eq. (71) one obtains [45]

$$
\begin{aligned}
\frac{\Delta h_{0}}{\hat{h}_{0}} \simeq & \left(0.06 \frac{q(q-1)}{2-q} \frac{L^{q} \mu_{0}}{2^{q-1} \hat{h}_{0}}\right)^{1 / q} \\
& \times\left(1.3 \times 10^{-15} \frac{T}{\hat{T}} \frac{\hat{\rho}}{\rho-\rho_{l}} \frac{\hat{r}^{3}}{r^{3}}\right)^{1-1 / q} .
\end{aligned}
$$

In comparison with Eq. (87), this theoretically required precision is considerably lower, especially for $q$ values close to unity. In contrast to this global precision $\Delta h_{0}$, the necessary local precision of the profile $h(y)$ may be a more serious problem in practice. Finally, we remark that the maximally enhanced diffusion in Eq. (64) and hence in Eq. (88) is not reached exactly at the critical tilt, cf. Eq. (66). The corresponding (negative) deviation from the critical $h_{0}$ value in Eq. (71) is of the same order of magnitude as $\Delta h_{0}$ in Eq. (89), see also Eq. (67).

\section{DISCUSSION}

In this paper, we have addressed the problem of overdamped Brownian motion in a tilted periodic potential in the presence of white thermal noise [31]. Our first main result is the compact and exact expression (22) for the diffusion coefficient, valid for arbitrary tilted periodic potentials and arbitrary strengths of the thermal noise. At the basis of this result lies Eq. (15), connecting the diffusion coefficient with the mean first passage time and the dispersion of the first passage times. The relations (14) and (15) have been previously proposed (without proof) in the context of random walk theory on discrete lattices [46], and they are also wellknown asymptotic relationships in the particular case of socalled renewal processes, see, e.g., formulas (14) and (16) in Chap. 5 of Ref. [47]. While all these works are concerned with models in discrete space, the use of the above relations for the continuous problem at hand has in fact been advocated (without proof) in Ref. [11], and an independent alternative derivation of Eq. (15), based on the results from Ref. [47], has recently been presented in Ref. [48]. Note also that a different expression for the diffusion coefficient (24) was recently proposed in Ref. [39], which is, in general, only approximatively valid (see Fig. 1 and Sec. IV). As a side remark we mention that our result has nothing to do with the relation between the diffusion coefficient and the Lyapunov exponent in certain chaotic dynamics [49]. In particular, the concept of Lyapunov exponents is useless in our context since it focuses on infinitesimal deviations between trajectories, while the diffusion coefficient here is governed by deviations ranging over many periods $L$.

It seem likely that a relation analogous to Eq. (15) can also be derived under more general conditions, e.g., in higher dimensions or beyond the overdamped limit $[39,50]$. While in these cases the mean first passage times and the dispersion of the passage times are generally not known in analytical form, such a relation may still be useful for speeding up the numerical determination of the diffusion coefficient. For spatially discrete, periodic, one-dimensional systems, an exact expression for the diffusion coefficient has been derived by Derrida in Ref. [51], see also Ref. [52] for various generalizations. It is clear that Derrida's result will become equivalent to Eq. (15) or Eq. (22) in the continuous space limit. Conversely, by considering periodic potentials $V_{0}(x)$ with very high barriers between neighboring local minima ("states"), an effective spatially discrete model is recovered. Again, it is clear that in this way our result will become equivalent to Derrida's discrete random walk result. In practice, our compact expression (22) for the diffusion coefficient is, however, considerably simpler than Derrida's.

Turning to our second main result, we recall that quantum mechanics is generally appreciated to be indispensable for the explanation, e.g., of the stability of atoms, molecules, and solids. Yet, verifying and exploiting basic quantum mechanical effects "more directly" on a macroscopic scale is currently attracting much attention. A somewhat similar situation arises with respect to the random microscopic fluctuations at the basis of statistical mechanics. As detailed in Sec. V, the present study suggests a very elementary experiment that would make those microscopic fluctuations "visible" on a macroscopic scale. In contrast to the quantum mechanical case, here the word "macroscopic" literally means "observable by the naked eye." 
We note that somewhat related phenomena are also known in other far-from-equilibrium systems. For example, the spreading of particles can be enhanced by the critical fluctuations in the vicinity of a nonequilibrium phase transition to a convective state, resulting in a diverging diffusion coefficient $D \sim\left(T-T_{c}\right)^{-z}$, where $z$ is a dynamical critical exponent [53]. Another well-known example is the enhancement of dispersion by turbulence. Diffusion can also be amplified by the coupling to a convective (laminar) process as exemplified in the well-studied example of Taylor dispersion with a characteristic diffusion coefficient given by $D \sim D_{0}^{-1}$ $\sim T^{-1}$, where $D_{0}$ is the usual (bare) molecular diffusion coefficient: see Ref. [54] for a review. In contrast to our present case, in those examples strong nonequilibrium fluctuations and/or gradients of the temperature and the velocity of the surrounding medium are a crucial ingredient. Two other examples that come closer to the situation of interest to us are, respectively, the Suzuki scaling law for relaxation from a marginally stable state [55] and the transient bistability in explosive systems [56]. In these cases, the dispersion is increased by the amplification of the initial thermal spreading in the close vicinity of a metastable point through the subsequent fast dynamical evolution away from this point. From this point of view, our present problem may be considered as the dispersion of particles in a potential displaying a spatially periodic repetition of marginally stable states-recall also our qualitative explanation of the effect below Eq. (55). Finally, we mention that a somewhat similar, resonancelike enhancement of the free thermal diffusion has also been reported for various systems in the presence of a timedependent external driving force [57-61]. While the behavior of the diffusion coefficient there is reminiscent of the present case, the underlying physical mechanisms are once again quite different.

\section{ACKNOWLEDGMENTS}

We gratefully acknowledge stimulating discussions with Markus Schreier, V. Baslakrishnan, Eli Pollak, Benjamin Lindner, and Lutz Schimansky-Geier. We are indebted to Benjamin Lindner and Lutz Schimansky-Geier for providing a copy of their working manuscript [48] prior to publication and for pointing out to us Ref. [47]. This work has been supported

by the exchange program of the Deutscher Akademischer Austauschdienst (P.R., P.H.) and the Acciones Integradas Hispano-Alemanas under HA1999-0081 (M.R., A.P.), the Program in Inter-University Attraction Poles of the Belgian Government and the F.W.O. Vlaanderen (C.V.d.B.), the Australian Research Council (H.L.), DFG-Sachbeihilfe HA1517/ 13-4 and the Graduiertenkolleg GRK283 (P.R., P.H.).

\section{APPENDIX A}

We present in this appendix some details pertinent to the derivation of Eq. (22). We first introduce the quantities

$$
\widetilde{I}_{+}(x):=\frac{1}{D_{0}} e^{V(x) / k T} \int_{-\infty}^{x} d y e^{-V(y) / k T}=\frac{I_{+}(x)}{1-e^{-L F / k T}},
$$

$$
\widetilde{I}_{-}(x):=\frac{1}{D_{0}} e^{-V(x) / k T} \int_{x}^{\infty} d y e^{V(y) / k T}=\frac{I_{-}(x)}{1-e^{-L F / k T}} .
$$

The last identity in Eq. (A1) follows from Eq. (17) and similarly for Eq. (A2). Next, we rewrite the first two moments in Eq. (10) as

$$
\begin{gathered}
T_{1}\left(x_{0} \rightarrow b\right)=\int_{x_{0}}^{b} d x \widetilde{I}_{+}(x)=\frac{1}{1-e^{-L F / k T}} \int_{x_{0}}^{b} d x I_{+}(x) \\
T_{2}\left(x_{0} \rightarrow b\right)= \\
\frac{2}{D_{0}} \int_{x_{0}}^{b} d x e^{V(x) / k T} \int_{-\infty}^{x} d y e^{-V(y) / k T} \\
\quad \times \int_{y}^{x} d z \widetilde{I}_{+}(z)+R
\end{gathered}
$$

Here $R$ is given by

$$
\begin{aligned}
R & :=\frac{2}{D_{0}} \int_{x_{0}}^{b} d x e^{V(x) / k T} \int_{-\infty}^{x} d y e^{-V(y) / k T} \int_{x}^{b} d z \widetilde{I}_{+}(z) \\
& =2 \int_{x_{0}}^{b} d x \widetilde{I}_{+}(x) \int_{x}^{b} d z \widetilde{I}_{+}(z) \\
& =2\left[T_{1}\left(x_{0} \rightarrow b\right)\right]^{2}-2 \int_{x_{0}}^{b} d x \widetilde{I}_{+}(x) \int_{a}^{x} d z \hat{I}_{+}(z) \\
& =2\left[T_{1}\left(x_{0} \rightarrow b\right)\right]^{2}-2 \int_{x_{0}}^{b} d x \widetilde{I}_{+}(z) \int_{z}^{b} d x \widetilde{I}_{+}(x) \\
& =2\left[T_{1}\left(x_{0} \rightarrow b\right)\right]^{2}-R=\left[T_{1}\left(x_{0} \rightarrow b\right)\right]^{2}
\end{aligned}
$$

Substituting this in Eq. (A4) we obtain

$$
\begin{aligned}
\Delta T_{2}\left(x_{0} \rightarrow b\right) & =\frac{2}{D_{0}} \int_{x_{0}}^{b} d x \int_{-\infty}^{x} d y \int_{y}^{x} d z e^{[V(x)-V(y)] / k T} \widetilde{I}_{+}(z) \\
& =\frac{2}{D_{0}} \int_{x_{0}}^{b} d x \int_{-\infty}^{x} d z \int_{-\infty}^{z} d y e^{[V(x)-V(y)] / k T} \widetilde{I}_{+}(z) \\
& =2 \int_{x_{0}}^{b} d x \int_{-\infty}^{x} d z e^{[V(x)-V(z)] / k T}\left[\widetilde{I}_{+}(z)\right]^{2} . \quad \text { (A6) }
\end{aligned}
$$

Using the fact that $\widetilde{I}_{+}(x)$ in Eq. (A1) is $L$ periodic, a similar calculation as in Eq. (17) then yields

$$
\begin{aligned}
& \Delta T_{2}\left(x_{0} \rightarrow b\right) \\
& \quad=\frac{2}{\left[1-e^{-L F / k T}\right]^{3}} \int_{x_{0}}^{b} d x \int_{x-L}^{x} d z e^{[V(x)-V(z)] / k T}\left[I_{+}(z)\right]^{2} .
\end{aligned}
$$


By choosing $b=x_{0}+L$ and interchanging the order of integration, we finally obtain

$$
\begin{aligned}
\Delta T_{2}\left(x_{0} \rightarrow x_{0}+L\right) & =\frac{2 D_{0}}{\left[1-e^{-L F / k T}\right]^{3}} \int_{x_{0}}^{x_{0}+L} d z\left[I_{+}(z)\right]^{2} I_{-}(z) \\
& =2 D_{0} \int_{x_{0}}^{x_{0}+L} d z\left[\widetilde{I}_{+}(z)\right]^{2} \widetilde{I}_{-}(z)
\end{aligned}
$$

With the definitions

$$
\begin{gathered}
H(x):=\int_{-\infty}^{x} d y e^{-V(y) / k T}, \\
K(x):=\int_{x}^{\infty} d y e^{V(y) / k T},
\end{gathered}
$$

we can rewrite Eq. (A3) as

$$
T_{1}\left(x_{0} \rightarrow x_{0}+L\right)=\frac{1}{D_{0}} \int_{x_{0}}^{x_{0}+L} d x\left[-K^{\prime}(x) H(x)\right] .
$$

Using the fact that the product $K(x) H(x)$ is $L$ periodic, a partial integration yields

$$
\begin{aligned}
T_{1}\left(x_{0} \rightarrow x_{0}+L\right) & =\frac{1}{D_{0}} \int_{x_{0}}^{x_{0}+L} d x H^{\prime}(x) K(x) \\
& =\int_{x_{0}}^{x_{0}+L} d x \widetilde{I}_{-}(x) \\
& =\frac{1}{1-e^{-L F / k T}} \int_{x_{0}}^{x_{0}+L} d x I_{-}(x)
\end{aligned}
$$

Similarly, Eq. (A8) can be rewritten as

$$
\begin{aligned}
\Delta T_{2}\left(x_{0} \rightarrow x_{0}+L\right)= & \frac{2}{D_{0}^{2}} \int_{x_{0}}^{x_{0}+L} d z \\
& \times\left[-K^{\prime}(z) H(z)\right]^{2} H^{\prime}(z) K(z) .
\end{aligned}
$$

Since $H^{\prime}(z) K^{\prime}(z)=1$ the integrand equals $(1 / 2) H^{2}(z) d K^{2}(z) / d z$; integration by parts yields

$$
\begin{aligned}
\Delta T_{2}\left(x_{0} \rightarrow x_{0}+L\right)= & \frac{2}{D_{0}^{2}} \int_{x_{0}}^{x_{0}+L} d z\left[-H^{\prime}(z) K(z)\right]^{2} \\
& \times\left[-K^{\prime}(z) H(z)\right] \\
= & 2 D_{0} \int_{x_{0}}^{x_{0}+L} d z\left[\widetilde{I}_{-}(z)\right]^{2} \widetilde{I}_{+}(z) \\
= & \frac{2 D_{0}}{\left[1-e^{-L F / k T}\right]^{3}} \int_{x_{0}}^{x_{0}+L} d z\left[I_{-}(z)\right]^{2} I_{+}(z) .
\end{aligned}
$$

Finally, using Eqs. (A3), (A8), (A12), and (A14) in Eq. (15) leads to the result (22).

\section{APPENDIX B}

In this appendix we discuss in some detail the special cases $q=2$ and $q=4 / 3$ that have been omitted in the main text in Sec. IV.

In the case $q=2$ we may choose, similarly as in the discussion below Eq. (43), a large value of $A$ and then approximately set $\epsilon=0$ and $A^{-q}=0$ in Eq. (35). The remaining integral does not converge for $a \rightarrow 0$ but we may introduce a convergence-inducing term according to

$$
\begin{aligned}
\int_{-L / 2}^{-a} d x I_{+}(x)= & \int_{-L / 2}^{-a} d x\left[\frac{\eta}{F_{c}-V_{0}^{\prime}(x)}-\frac{\eta}{2 \mu|x|}\right] \\
& +\int_{-L / 2}^{-a} d x \frac{\eta}{2 \mu|x|} .
\end{aligned}
$$

The first integral converges for $a \rightarrow 0$ and by actually performing this limit we make an error that vanishes for $k T$ $\rightarrow 0$, see Eq. (34). The second integral in Eq. (B1) can be performed, with the result

$$
\begin{aligned}
\int_{-L / 2}^{-a} d x I_{+}(x)= & \int_{-L / 2}^{0} d x\left[\frac{\eta}{F_{c}-V_{0}^{\prime}(x)}-\frac{\eta}{2 \mu|x|}\right] \\
& +\frac{\eta}{2 \mu}\left[\frac{1}{2} \ln \left(\frac{L F_{c}}{2 k T}\right)+\ln \left(\frac{\mu L}{2 F_{c}}\right)-\ln A\right]
\end{aligned}
$$

where we have used Eq. (34). Exactly the same result is recovered for Eq. (36). Turning to Eq. (38), a convergence inducing term for $A \rightarrow \infty$ may be introduced according to

$$
\begin{aligned}
\int_{-a}^{a} d x I_{+}(x)= & \frac{\eta}{\mu} \int_{-A}^{A} d x\left[K(x, \gamma)-\frac{1}{2} \frac{1}{1+|x|}\right] \\
& -\frac{\eta}{\mu} \int_{-A}^{A} d x \frac{1}{2} \frac{1}{1+|x|} .
\end{aligned}
$$

Indeed, the first integral now converges when $A \rightarrow \infty$, while the second one can be performed. Neglecting all contributions that tend to zero when $A \rightarrow \infty$, we thus obtain

$$
\int_{-a}^{a} d x I_{+}(x)=\frac{\eta}{\mu} \int_{-\infty}^{\infty} d x\left[K(x, \gamma)-\frac{1}{2} \frac{1}{1+|x|}\right]-\frac{\eta}{\mu} \ln A .
$$

Putting together everything, we finally find that

$$
\int_{x_{0}}^{x_{0}+L} d x I_{ \pm}(x)=\frac{\eta}{\mu}\left[\frac{1}{2} \ln \left(\frac{F_{c} L}{2 k T}\right)+\widetilde{G}_{1}(\gamma)+C\right] \text { for } q=2,
$$

where we have introduced 


$$
\begin{gathered}
\widetilde{G}_{1}(\gamma):=\int_{-\infty}^{\infty} d x\left[K(x, y)-\frac{1}{2} \frac{1}{1+|x|}\right], \\
C:=\ln \left(\frac{\mu L}{2 F_{c}}\right)+\int_{-L / 2}^{L / 2} d x\left[\frac{\mu}{F_{c}-V_{0}^{\prime}(x)}-\frac{1}{2|x|}\right] .
\end{gathered}
$$

As in the case of $G_{1}(\gamma)$ in Eq. (42), $\widetilde{G}_{1}(\gamma)$ is again a universal, dimensionless scaling function of its dimensionless argument $\gamma$. Further, the saddle point approximation (46) can be applied for large negative $\gamma$ values. Finally, $C$ is a constant of order unity that depends on the details of the potential $V_{0}(x)$. For instance, when the form (26) is exactly valid on the entire interval $[-L / 2, L / 2]$ then we have $C=0$.

A similar calculation yields the result

$$
\begin{gathered}
\int_{x_{0}}^{x_{0}+L} d x I_{ \pm}(x) I_{+}(x) I_{-}(x)=\frac{81}{128}\left(\frac{\eta}{\mu}\right)^{3} \ln \left(\frac{F_{c} L}{2 k T}\right) \\
\text { for } q=4 / 3,
\end{gathered}
$$

where we have restricted ourselves to the leading order term in the weak noise limit $k T / F_{c} L \rightarrow 0$, i.e., higher order terms analogous to those on the right-hand side of Eq. (B5) have been omitted.

For the resulting diffusion coefficient (22) one obtains

$$
D=D_{0} \frac{L^{2} \mu}{k T} \frac{G_{3}(\gamma)}{\left[\frac{1}{2} \ln \left(\frac{F_{c} L}{2 k T}\right)+\widetilde{G}_{1}(\gamma)+C\right]^{3}} \text { for } q=2 \text {, }
$$

$$
D=D_{0} \frac{\frac{81}{128} \ln \left(\frac{F_{c} L}{2 k T}\right)}{\left[\int_{x_{0}}^{x_{0}+L} \frac{d x \mu L^{-2 / 3}}{F_{c}-V_{0}^{\prime}(x)}\right]^{3}} \text { for } q=4 / 3
$$

The detailed discussion of the result (B9) can be carried out in complete analogy to the one for $2>q>4 / 3$ below Eq. (55). In particular, for sufficiently small $k T$, the second and third term in the denominator are negligible for positive and moderately negative $\gamma$ values, while for large negative $\gamma$ the saddle point approximation (46) for $\widetilde{G}_{1}(\gamma)$ and $G_{3}(\gamma)$ $=[S(\gamma)]^{2} / 2$ can be applied. In Eq. (B10) only the leading order terms for small $k T$ have been kept. Thus the discussion of the dependence for a fixed $k T$ upon variation of $\gamma$ is not possible on the basis of Eq. (B10) but it is clear that this dependence will be of exactly the same form as the one for 4/3>q>1 discussed below Eq. (68). Especially, Eqs. (64)(68) can be taken over for $q=4 / 3$ without any change.
[1] A. Einstein, Ann. Phys. (Leipzig) 17, 549 (1905).

[2] S. Lifson and J. L. Jackson, J. Chem. Phys. 36, 2410 (1962).

[3] L. P. Kadanoff, Physica A 163, 1 (1990).

[4] H. Fujisaka and S. Grossmann, Z. Phys. B: Condens. Matter 48, 261 (1982).

[5] T. Geisel and J. Nierwetberg, Phys. Rev. Lett. 48, 7 (1982).

[6] M. Schell, S. Fraser, and R. Kapral, Phys. Rev. A 26, 504 (1982).

[7] T. Geisel and J. Nierwetberg, Z. Phys. B: Condens. Matter 56, 59 (1984).

[8] R. Klages and J. R. Dorfman, Phys. Rev. Lett. 74, 387 (1995).

[9] L. S. Tsimring, Physica D 63, 41 (1993).

[10] P. Reimann, Phys. Rev. E 50, 727 (1994).

[11] P. Reimann and C. Van den Broeck, Physica D 75, 509 (1994).

[12] D. Sigeti and W. Horsthemke, J. Stat. Phys. 54, 1217 (1989).

[13] S. Strogatz, Nonlinear Dynamics and Chaos (Addison-Wesley, Reading, 1994).

[14] H. Risken, The Fokker-Planck Equation (Springer, Berlin, 1984).

[15] A. Barone and G. Paterno, Physics and Applications of the Josephson Effect (Wiley, New York, 1982).

[16] B. Shapiro, I. Dynan, M. Gitterman, and G. H. Weiss, Phys. Rev. B 46, 8416 (1992).

[17] D. Reguera, J. M. Rubi, and A. Pérez-Madrid, Phys. Rev. E 62, 5313 (2000).

[18] Y. Georgievskii and A. Burshtein, J. Chem. Phys. 100, 7319 (1994).

[19] P. Fulde, L. Pietronero, W. R. Schneider, and S. Strässler, Phys. Rev. Lett. 35, 1776 (1975); W. Dietrich, P. Fulde, and I. Peschel, Adv. Phys. 29, 527 (1980).
[20] G. Gruner, A. Zawadowski, and P. M. Chaikin, Phys. Rev. Lett. 46, 511 (1981).

[21] R. Adler, Proc. IRE 34, 351 (1946); I. I. Fedchina and N. A. Usova, Z. Phys. B: Condens. Matter 50, 263 (1983); 52, 69 (1983); P. Hänggi and P. Riseborough, Am. J. Phys. 51, 347 (1983).

[22] W. C. Lindsey, Synchronization Systems in Communication and Control (Prentice Hall, Englewood Cliffs, NJ, 1972).

[23] W. Schleich, C. S. Cha, and J. D. Cresser, Phys. Rev. A 29, 230 (1984).

[24] T. Katsouleas and J. Dawson, Phys. Rev. Lett. 51, 392 (1983).

[25] J. W. M. Frenken and J. F. Van der Veen, Phys. Rev. Lett. 54, 134 (1985); B. Pluis et al., ibid. 59, 2678 (1987); D. C. Senft and G. Ehrlich, ibid. 74, 294 (1995); T. R. Linderoth, S. Horch, E. Laegsgaard, I. Stensgaard, and F. Besenbacher, ibid. 78, 4978 (1997); P. Talkner, E. Hershkovitz, E. Pollak, and P. Hänggi, Surf. Sci. 437, 198 (1999).

[26] A. Ajdari and J. Prost, Proc. Natl. Acad. Sci. U.S.A. 88, 4468 (1992); G. I. Nixon and G. W. Slater, Phys. Rev. E 53, 4969 (1996); C. L. Asbury and G. van den Engh, Biophys. J. 74, 1024 (1998).

[27] F. C. Hoppenstead and E. M. Izhikevich, Weakly Connected Neural Networks (Springer, New York, 1974), p. 74; K. Wiesenfeld, D. Pierson, E. Pantazelou, C. Dames, and F. Moss, Phys. Rev. Lett. 72, 2125 (1994); C. Kurrer and K. Schulten, Phys. Rev. E 51, 6213 (1995).

[28] For a review see: P. Reimann, e-print cond-mat/0010237.

[29] M. E. Shaposhnikov (private communication); and Contemp. Phys. 39, 177 (1998).

[30] M. Borromeo and F. Marchesoni, Phys. Lett. A 249, 8457 
(1998); C. Van den Broeck, Europhys. Lett. 46, 1 (1999); R. Landauer and M. Büttiker, Phys. Scr. T9, 155 (1985); L. P. Faucheux, G. Stolovitzky, and A. Libchaber, Phys. Rev. E 51, 5239 (1995); V. I. Talyanskii, J. M. Shilton, M. Pepper, C. G. Smith, C. J. B. Ford, E. H. Linfield, D. A. Ritchie, and G. A. C. Jones, Phys. Rev. B 56, 15180 (1997); C. Rocke, S. Zimmermann, A. Wixforth, J. P. Kotthaus, G. Böhm, and G. Weimann, Phys. Rev. Lett. 78, 4099 (1997).

[31] P. Reimann, C. Van den Broeck, H. Linke, P. Hänggi, M. Rubi, and A. Pérez-Madrid, Phys. Rev. Lett. 87, 010602 (2001).

[32] P. Hänggi, P. Talkner, and M. Borkovec, Rev. Mod. Phys. 62, 251 (1990).

[33] R. L. Stratonovich, Radiotekh. Elektron. (Moscow) 3, 497 (1958). English translation in Non-Linear Transformations of Stochastic Processes, edited by P. I. Kuznetsov, R. L. Stratonovich, and V. I. Tikhonov (Pergamon, Oxford, 1965).

[34] Einstein's original derivation yields Eq. (7) for $F=0$. The case $F \neq 0$ can be readily reduced to the case $F=0$ by means of the transformation $x(t) \mapsto x(t)-\eta F t$. Since this transformation leaves the diffusion coefficient (6) invariant, one recovers Eq. (7) for arbitrary values of the static tilt $F$ in Eq. (1).

[35] A more rigorous derivation can be found, e.g., in Ref. [62]. The same result follows immediately from the fluctuationdissipation relation $D=k T d\langle\dot{x}\rangle /\left.d F\right|_{F=0}$ in combination with Stratonovich's formula (18).

[36] Differently speaking, this is so because Eq. (1) is a so-called strong Markov process, and first passage times are so-called Markov stopping times.

[37] We emphasize that the limit $t \rightarrow \infty$ in Eqs. (5) and (6) is associated with $m \rightarrow \infty$, not with $l \rightarrow \infty$. In other words, our line of reasoning goes as follows: First, one chooses a large $l$, which is then kept fixed. Next, one lets $m \rightarrow \infty$ and thus $t \rightarrow \infty$. As a result, one obtains relations like in Eqs. (14) and (15) but in addition with certain finite $l$ corrections. Finally, by choosing a sufficiently large $l$, these corrections become arbitrarily small. In particular, the two limits $l \rightarrow \infty$ and $m \rightarrow \infty$ (and thus $t \rightarrow \infty$ ) are never interchanged.

[38] Due to this fact that the transitions between "states" are unidirectional and independent, identically distributed random events, the coarse-grained counterpart of $x(t)$ is a so-called renewal process with the first passage time distribution between subsequent positions playing the role of the waiting time distribution or renewal function [47]. Keeping in mind that the current $\langle\dot{x}\rangle$ and the diffusion coefficient $D$ are identical for the original process $x(t)$ and its coarse-grained counterpart, those quantities can now be inferred [48] by exploiting well-known results from the theory of renewal processes. Indeed, Eqs. (14) and (15) are well-known asymptotic relationships in the theory of renewal processes, see, e.g., formulas (14) and (16) in Chapter 5 of Ref. [47]. In our present paper, we provide an alternative, self-contained derivation of these relations.

[39] G. Constantini and F. Marchesoni, Europhys. Lett. 48, 491 (1999).

[40] A. W. Hübler, Ph.D. thesis, München, 1987.

[41] In the context of chromatography, $Q$ from Eq. (70) is called the retention parameter, while $1 / Q$ is called Perclet number.

[42] J. Happel and H. W. Brenner, Low Reynolds Number Hydrodynamics with Special Applications to Particulate Media (Prentice-Hall, Engelwood Cliffs, NJ, 1965).

[43] As an alternative experimental implementation one may imagine that the particle is actually a pearl, moving along a very thin but rigid wire. While such a setup may be somewhat academic it has the conceptual advantage that all the correction effects in Eq. (78) are then indeed negligible and the constraint $z=h(y)$ in place of $z \geqslant h(y)$ is strictly valid.

[44] For a somewhat related experimental investigation see also Ref. [40].

[45] This result (89) follows by exploiting $\Delta F=\Delta h_{0} F_{g} / L$ [cf. Eq. (76)], $\Delta \gamma=\Delta F \mu^{-1 / q}[k T]^{1 / q-1} \quad$ [cf. Eq. (39)], and Eqs. (66), (67).

[46] M. Khantha and V. Balakrishnan, Pramana 21, 111 (1983); C. Van den Broeck, in Noise and Nonlinear Phenomena in Nuclear Systems, edited by J. L. Munoz-Cobo and F. C. Difilippo (Plenum, New York, 1989), p. 3.

[47] D. R. Cox, Renewal Theory (Methuen, London, 1962).

[48] B. Lindner, M. Kostur, and L. Schimansky-Geier, Fluct. Noise Lett. 1, R25 (2001).

[49] P. Gaspard and G. Nicolis, Phys. Rev. Lett. 65, 1693 (1990).

[50] M. Borromeo and F. Marchesoni, Phys. Rev. Lett. 84, 203 (2000).

[51] B. Derrida, J. Stat. Phys. 31, 433 (1983).

[52] I. Claes and C. Van den Broeck, J. Stat. Phys. 70, 1215 (1993); Z. Koza, J. Phys. A 32, 7637 (1999).

[53] H. N. W. Lekkerkerker, Physica A 80, 415 (1975).

[54] C. Van den Broeck, Physica A 168, 677 (1990).

[55] M. Suzuki, in Proceedings of the XVII Solvay Conference on Physics, edited by G. Nicolis, G. Dewel, and J. W. Turner (Wiley, New York, 1981), p. 299.

[56] G. Nicolis and F. Baras, J. Stat. Phys. 48, 1071 (1987).

[57] I. Claes and C. Van den Broeck, Phys. Rev. A 44, 4970 (1991).

[58] A. Ghosh, Phys. Lett. A 187, 54 (1994).

[59] H. Gang, A. Daffertshofer, and H. Haken, Phys. Rev. Lett. 76, 4874 (1996).

[60] M. Schreier, P. Reimann, P. Hänggi, and E. Pollak, Europhys. Lett. 44, 416 (1998).

[61] Y. Kim and W. Sung, Phys. Rev. E 57, R6237 (1998).

[62] R. Festa and E. Galleani d'Agliano, Physica A 90, 229 (1978). 